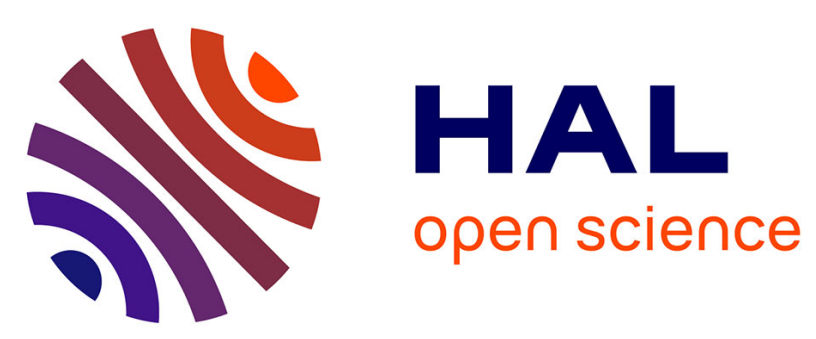

\title{
Apport des stalagmites pour l'étude de la grotte Chauvet: datations absolues U/Th (TIMS) et reconstitution paléoclimatique par les isotopes stables de la calcite
}

Dominique Genty, Dominique Blamart, Bassam Ghaleb

\section{To cite this version:}

Dominique Genty, Dominique Blamart, Bassam Ghaleb. Apport des stalagmites pour l'étude de la grotte Chauvet: datations absolues U/Th (TIMS) et reconstitution paléoclimatique par les isotopes stables de la calcite. Bulletin de la Société préhistorique française, 2005, 102 (1), pp.45-62. 10.3406/bspf.2005.13336 . hal-02965738

\section{HAL Id: hal-02965738 \\ https://hal.science/hal-02965738}

Submitted on 18 Feb 2021

HAL is a multi-disciplinary open access archive for the deposit and dissemination of scientific research documents, whether they are published or not. The documents may come from teaching and research institutions in France or abroad, or from public or private research centers.
L'archive ouverte pluridisciplinaire HAL, est destinée au dépôt et à la diffusion de documents scientifiques de niveau recherche, publiés ou non, émanant des établissements d'enseignement et de recherche français ou étrangers, des laboratoires publics ou privés.

\section{(ㅇ)(1) $\$$}

Distributed under a Creative Commons Attribution - NonCommercial - NoDerivatives 44.0 
Apport des stalagmites pour l'étude de la grotte Chauvet : datations absolues U/Th (TIMS) et reconstitution paléoclimatique par les isotopes stables de la calcite

Dominique Genty, Dominique Blamart, Bassam Ghaleb

\section{Citer ce document / Cite this document :}

Genty Dominique, Blamart Dominique, Ghaleb Bassam. Apport des stalagmites pour l'étude de la grotte Chauvet : datations absolues U/Th (TIMS) et reconstitution paléoclimatique par les isotopes stables de la calcite. In: Bulletin de la Société préhistorique française, tome 102, $\mathrm{n}^{\circ} 1,2005$. La grotte Chauvet à Vallon-Pont-d'Arc : un bilan des recherches pluridisciplinaires Actes de la séance de la Société préhistorique française, 11 et 12 octobre 2003, Lyon. pp. 45-62; doi : https://doi.org/10.3406/bspf.2005.13336

https://www.persee.fr/doc/bspf_0249-7638_2005_num_102_1_13336

Fichier pdf généré le 10/01/2019 


\begin{abstract}
A first set of speleothems (stalagmites and flow stone s) from the Chauvet Cave (Ardèche, southern France) were dated using U/Th and $14 \mathrm{C}$ mass spectrométrie methods. At the same time, stable isotope analyses $(\delta 13 \mathrm{C}$ and $\delta 180)$ were done on several stalagmites and on seepage water and rainfall $(\delta 180$ and $\delta D)$. These results give the following information: firstly, accurate ages of several geomorphological events that have marked the cave's history since $34 \mathrm{kyr}$ and, secondly, precisions concerning the climatic evolution in this area since the last déglaciation. Thus, several events have been recorded and dated: the last déglaciation, the Bolling- Allerød (BA) and the Younger Dryas (YD). A warm and short climatic event was detected during the YD itself around $12.15 \mathrm{kyrs}$. The Chauvet Cave speleothems appear to be exceptional recorders of continental palaeoenvironmental variations similar, in time resolution and in climate sensitivity, to ice, marine or lake records for the same period, with the advantage of an absolute chronology.
\end{abstract}

\title{
Résumé
}

Un premier ensemble de stalagmites et de planchers stalagmitiques prélevés dans la grotte Chauvet (Vallon-Pont-d'Arc, Ardèche) a été daté par des méthodes radiométriques par spectrométrie de masse (U/Th et 14C). Parallèlement, des analyses d'isotopes stables ( $\delta 13 \mathrm{C}$ et $\delta 180$ ) ont été effectuées sur plusieurs stalagmites et sur les eaux d'infiltration et les eaux de pluie ( $\delta 180$ et $\delta D$ ). Ces résultats apportent, d'une part, des précisions sur la chronologie des divers événements qui ont marqué I'histoire géomorphologique de la grotte depuis 34 ha, et, d'autre part, ils permettent de reconstituer l'environnement climatique qui a perduré pendant la formation de ces stalagmites. Ainsi, la dernière déglaciation, le Bolling-Allerød (BA) et le Younger-Dryas (YD), a été mise en évidence de façon remarquable. Un événement climatique chaud et de courte durée a aussi été mis en évidence autour de 12, $15 \mathrm{ka}$, en plein milieu du YD. Les stalagmites de la grotte Chauvet constituent ainsi un enregistrement des variations environnementales continentales exceptionnel, comparable, dans sa résolution temporelle et dans sa sensibilité climatique, aux enregistrements glaciaires, marins ou lacustres de la même période, avec l'avantage, ici, d'avoir une chronologie absolue. 


\title{
Apport des stalagmites pour l'étude de la grotte Chauvet : datations absolues U/Th (TIMS) et reconstitution paléoclimatique par les isotopes stables de la calcite
}

\begin{abstract}
Résumé
Un premier ensemble de stalagmites et de planchers stalagmitiques prélevés dans la grotte Chauvet (Vallon-Pont-d'Arc, Ardèche) a été daté par des méthodes radiométriques par spectrométrie de masse $\left(U / T h\right.$ et $\left.{ }^{14} \mathrm{C}\right)$. Parallèlement, des analyses d'isotopes stables $\left(\delta^{13} \mathrm{C}\right.$ et $\left.\delta^{18} \mathrm{O}\right)$ ont été effectuées sur plusieurs stalagmites et sur les eaux d'infiltration et les eaux de pluie $\left(\delta^{18} O\right.$ et $\delta D$ ). Ces résultats apportent, d'une part, des précisions sur la chronologie des divers événements qui ont marqué l'histoire géomorphologique de la grotte depuis $34 \mathrm{ka}$, et, d'autre part, ils permettent de reconstituer l'environnement climatique qui a perduré pendant la formation de ces stalagmites. Ainsi, la dernière déglaciation, le Bølling-Allerød (BA) et le Younger-Dryas (YD), a été mise en évidence de façon remarquable. Un événement climatique chaud et de courte durée a aussi été mis en évidence autour de $12,15 \mathrm{ka}$, en plein milieu du YD. Les stalagmites de la grotte Chauvet constituent ainsi un enregistrement des variations environnementales continentales exceptionnel, comparable, dans sa résolution temporelle et dans sa sensibilité climatique, aux enregistrements glaciaires, marins ou lacustres de la même période, avec l'avantage, ici, d'avoir une chronologie absolue.
\end{abstract}

\begin{abstract}
A first set of speleothems (stalagmites and flowstones) from the Chauvet Cave (Ardèche, southern France) were dated using U/Th and ${ }^{14} \mathrm{C}$ mass spectrometric methods. At the same time, stable isotope analyses $\left(\delta^{13} \mathrm{C}\right.$ and $\left.\delta^{18} O\right)$ were done on several stalagmites and on seepage water and rainfall $\left(\delta^{18} O\right.$ and $\delta D$ ). These results give the following information: firstly, accurate ages of several geomorphological events that have marked the cave's history since $34 \mathrm{kyr}$ and, secondly, precisions concerning the climatic evolution in this area since the last deglaciation. Thus, several events have been recorded and dated: the last deglaciation, the BøllingAllerod (BA) and the Younger Dryas (YD). A warm and short climatic event was detected during the YD itself around 12.15 kyrs. The Chauvet Cave speleothems appear to be exceptional recorders of continental palaeoenvironmental variations similar, in time resolution and in climate sensitivity; to ice, marine or lake records for the same period, with the advantage of an absolute chronology.
\end{abstract}


Fig. 1 - Localisation des échantillons et des stations d'échantillonnage d'eau d'infiltration (bleu) (base topographique : Y. Le GuillouF. Maksud. En hommage à François Rouzaud)

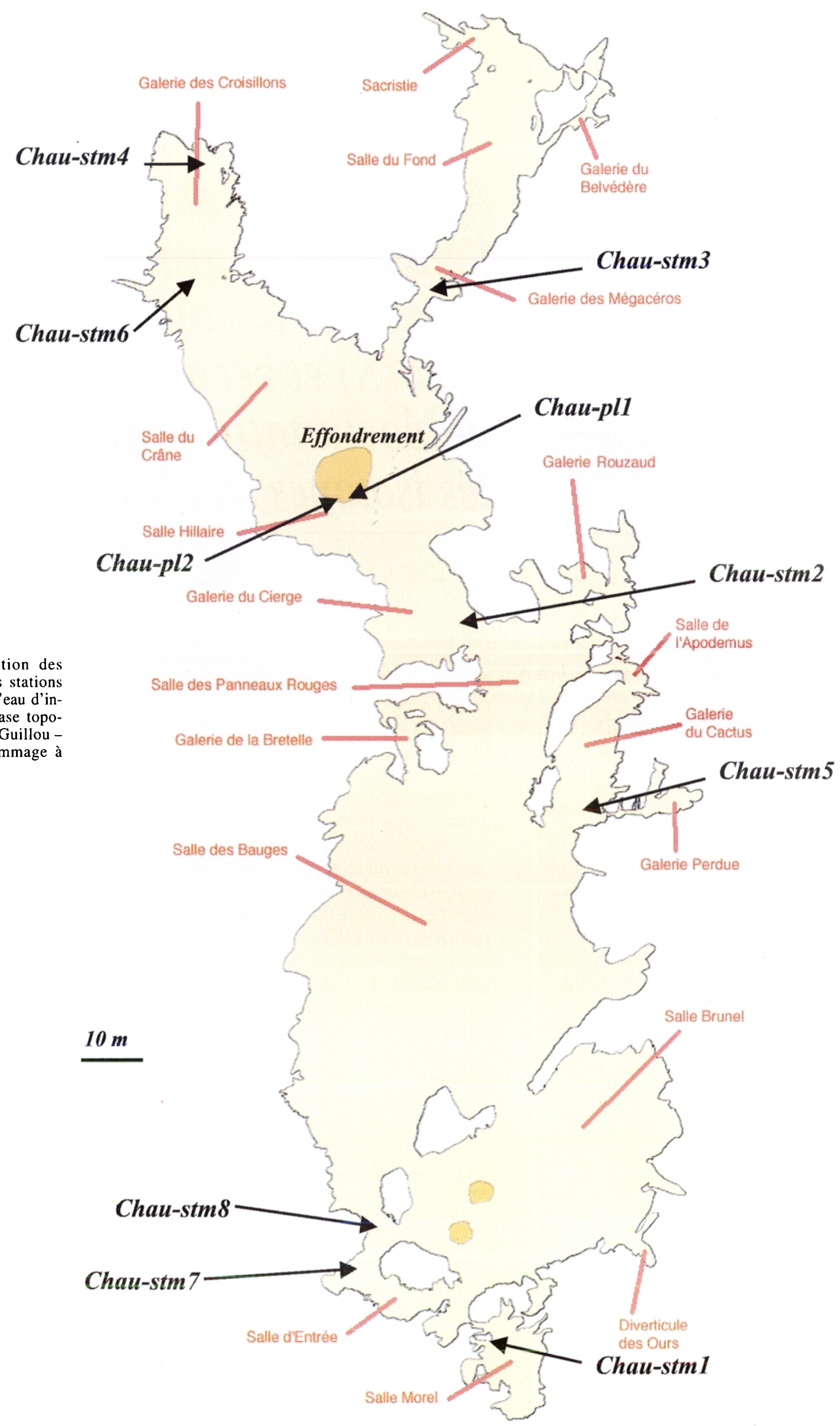




\section{INTRODUCTION}

La grotte Chauvet, avant d'avoir été visitée par l'homme, a eu une histoire géologique complexe : sa formation et une partie de ses remplissages résultent des variations du niveau de base de l'Ardèche (Delannoy et al., 2001 et 2003), située maintenant $100 \mathrm{~m}$ en contrebas, et la succession de ses dépôts stalagmitiques résulte aussi, en partie, des variations climatiques marquées du Quaternaire. L'aspect actuel de la grotte présente plusieurs caractéristiques : un éboulis proche de la falaise obstruant l'entrée d'origine, de vastes salles dont le sol est en grande partie recouvert de planchers stalagmitiques et de stalagmites, d'effondrements à l'emporte-pièce laissant apparaître les remplissages détritiques sous les planchers stalagmitiques (fig. 1). S'il n'y a pas de moyen direct de dater les premières phases des dépôts dans la grotte, il est par contre possible, grâce aux datations uranium-thorium (U/Th) faites sur les stalagmites et les planchers stalagmitiques, de donner des jalons chronologiques à l'histoire la plus récente de la grotte (la limite théorique des datations U/Th par spectrométrie de masse par thermo-ionisation (TIMS) est d'environ $500 \mathrm{ka} ; \mathrm{Li}$ et al., 1989). Nous présentons ici un premier ensemble de dates U/Th (TIMS) et ${ }^{14} \mathrm{C}$ (AMS) effectuées sur huit stalagmites et deux planchers stalagmitiques dont l'âge remonte à $\sim 34 \mathrm{ka}$. Ces échantillons, prélevés à proximité des passerelles, permettent de postdater (donner un âge minimum) l'éboulis d'entrée, les sols archéologiques qu'ils scellent (et indirectement les charbons qu'ils renferment) et l'effondrement de la salle Hillaire (fig. 1). La plupart de ces résultats sont détaillés dans Genty et al. (2004), nous en rappelons ici les principaux. Parallèlement à ces résultats chronologiques, nous présentons ici une reconstitution paléoclimatique partielle des derniers 34 ka basée sur les isotopes stables de la calcite des stalagmites $\left(\delta^{13} \mathrm{C}\right.$ et $\left.\delta^{18} \mathrm{O}\right)$ et sur les vitesses de croissance de ces concrétions.

\section{LOCALISATION ET DESCRIPTION DES ÉCHANTILLONS}

L'échantillonnage des concrétions a été contraint, d'une part, par le parcours du cheminement mis en place dès la découverte de la grotte (Chauvet et al., 1995) et, d'autre part, par le souci de préserver l'aspect esthétique du site. Huit stalagmites et deux morceaux de planchers stalagmitiques ont été prélevés dans la grot te (fig. 1; tabl. 1 et 2). Ils sont tous composés de calcite ayant une fabrique cristalline palissadique typique : cristaux millimétriques à centimétriques perpendiculaires aux lamines de croissance (Kendall et al. 1978). Il existe, au sein même des échantillons. des variations dans la porosité de la calcite donnant deux faciès bien distincts : la calcite compacte et translucide et la calcite poreuse et blanche. Ces deux faciès traduisent des changements dans les conditions de précipitation du carbonate (Genty et Quinif. 1996). Par exemple, dans la stalagmite Chau-stm4. une variation brusque de ce type montre qu un événement a modifié les conditions de précipitation il y a 4950 ans $\pm 75($ ) (âge ${ }^{14} \mathrm{C}$ corrigé du carbone mort et calibré; photo 1 ). Un tel changement peut donner des indications sur les changements d'environnement; ainsi, la calcite poreuse a tendance à se former dans des conditions de forte sursaturation (évaporation ou dégazage de $\mathrm{CO}_{2}$ intense); elle est souvent liée à une croissance rapide, alors que la calcite compacte correspond souvent à une croissance et à un dégazage de $\mathrm{CO}_{2}$ plus lents (Frisia et al., 2000; Genty et Quinif, 1996). Il existe aussi des fabriques poreuses liées à un film d'eau (qui recouvre le sommet de la stalagmite) plus épais. Le passage d'une calcite blanche et poreuse à une calcite compacte sur la stalagmite Chau-stm4 peut donc être lié à des

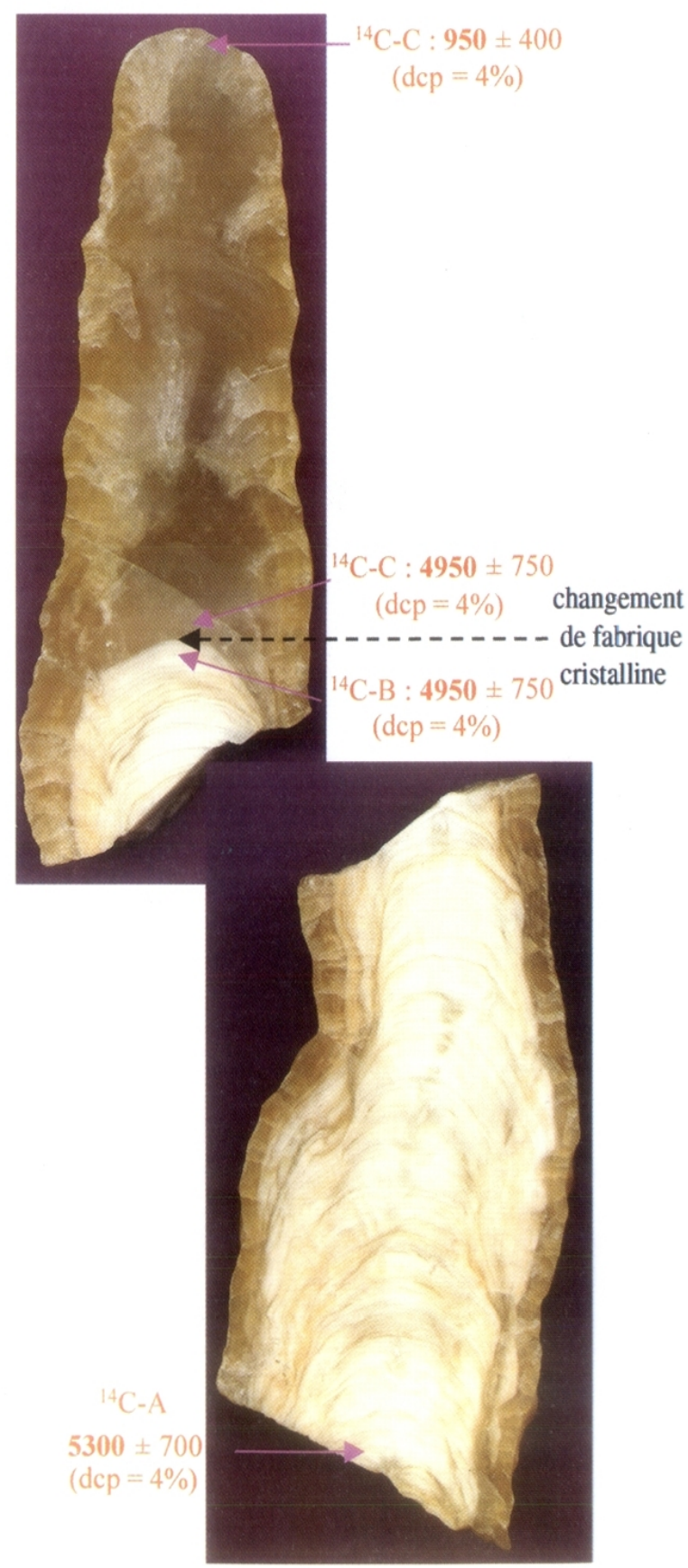

Photo 1 - Section verticale polie d'une partic de la stalagmite Chau-stmmontrant un changement de faciès de la calcite: WPC white porous (alcite') et I)(' (dark compact calcite'). 


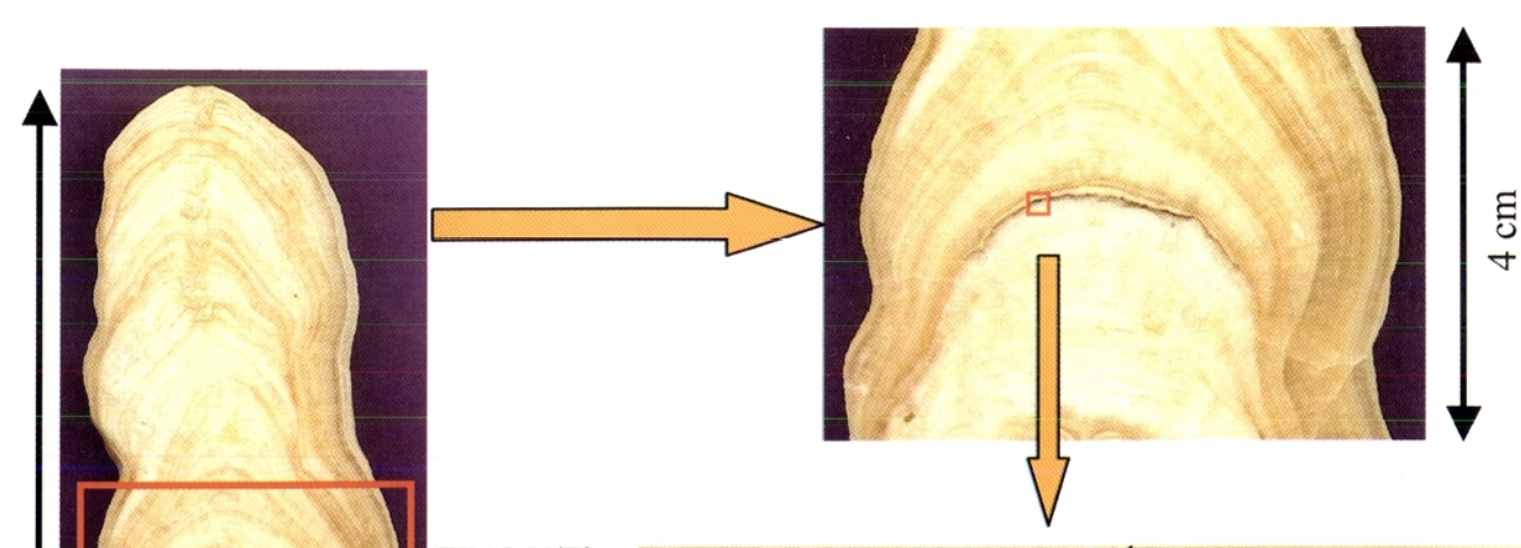

U
J

TIMS U/Th :

$3600 \pm 85 \mathrm{yr}$

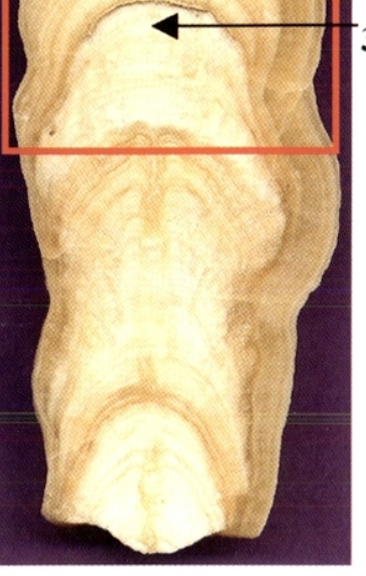

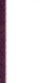

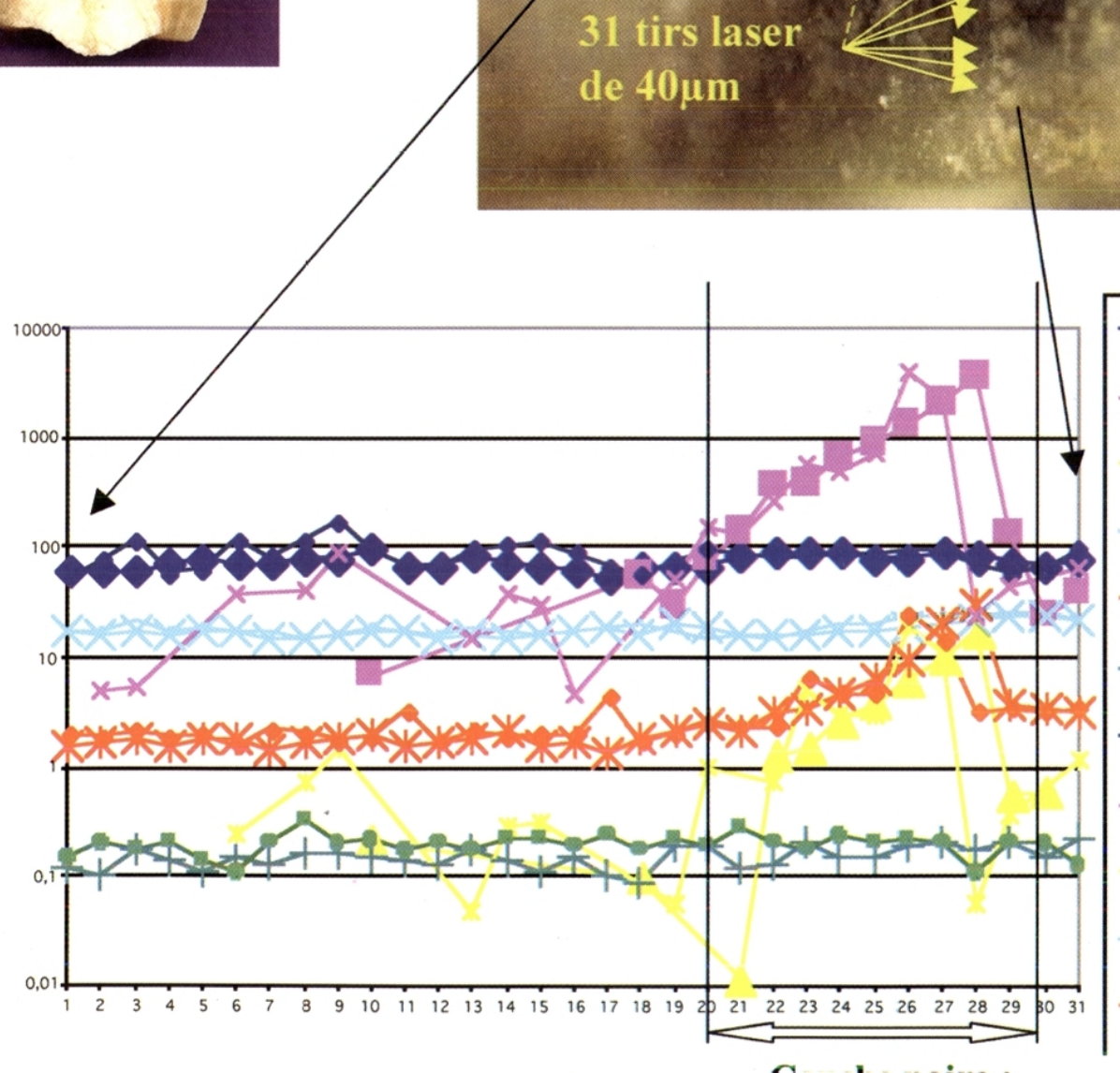

31 tirs laser

\section{de $40 \mu \mathrm{m}$}

\begin{tabular}{|c|}
- Magnésium \\
Cobalt \\
- Strontium \\
- Banganèse \\
- Uranium \\
- Magnésium \\
Cobalt \\
Strontium \\
Banganèse
\end{tabular}

Couche noire :

$\mathrm{Mn}, \mathrm{Co}, \mathrm{Ba}$

Fig. 2 - Analyse d'une couche noire de la stalagmite Chau-stm 1 par ablation laser et ICPMS. Trente et un tirs laser de $40 \mu \mathrm{m}$ de diamètre ont permis de caractériser la couche noire de Chau-stm1 : celle-ci, marquée par une augmentation dans les concentrations de Mn, Co, Ba, est composée d'oxyde de Mn liée à une décharge naturelle. Analyses effectuées au musée de Thervuren (Bruxelles, Belgique) par J. Navez et D. Genty, en collaboration avec E. Keppens, S. Verheyden. 
conditions de précipitation de la calcite plus confinées, ou à l'arrivée d'eau moins sursaturée ou à une réduction du débit. Cependant, la signification environnementale des différentes fabriques cristallines, du fait de trop rares études (en particulier sur les calcites actuelles dans différents environnements), reste qualitative et à considérer avec précaution; elle peut seulement apporter une confirmation à d'autres paramètres géochimiques mesurés en parallèle comme les isotopes stables ou les teneurs en éléments mineurs.

Au cours de leur croissance, les stalagmites piègent de nombreux restes aussi divers que des insectes (des pattes d'insectes ont été retrouvées dans une stalagmite de la grotte de Villars, Dordogne; Genty et al., 2001), des fragments de végétaux (de nombreux poils de végétaux aussi), des particules de charbon liées à l'activité humaine en surface (Perrette et al., 1997), des fragments de torche de paille liées à des visites (Genty et al., 1997). Il est donc concevable que des pigments et des particules liées à l'exécution des peintures pariétales puissent aussi se piéger dans les stalagmites; l'intérêt serait alors de pouvoir dater indirectement cette activité humaine en datant la calcite. C'est dans ce but que nous avons analysé une couche noire repérée dans la stalagmite Chau-stml dès son sciage alors que son âge était encore inconnu. La technique utilisée, l'ablation laser couplée à un spectromètre de masse à plasma induit (LA-ICP-MS) est quasi non destructrice et permet de doser plusieurs dizaines d'éléments simultanément. Un profil d'une trentaine de tirs laser de $40 \mu \mathrm{m}$ de diamètre a été effectué perpendiculairement à cette couche noire (fig. 2). Par rapport à la calcite qui l'entoure, ce dépôt se caractérise par une augmentation des concentrations en $\mathrm{Mn}$, Co et $\mathrm{Ba}$. Ces éléments entrent dans la composition des oxydes de manganèse, terme générique qui en fait regroupe de nombreuses variétés minéralogiques. Celles-ci ont été étudiées en détail dans les grottes ornées par microsonde électronique et diffractomérie X (Vouvé, 1995; Menu et Walter, 1995; Baujard, 2000; etc.). L'origine de la couche noire observée dans Chau-stml est probablement liée à un apport naturel par les eaux d'infiltration qui, en traversant la zone non saturée, ont libéré une poche d'oxyde de manganèse.

\section{MÉTHODES EXPÉRIMENTALES}

Les méthodes U/Th et ${ }^{14} \mathrm{C}$ utilisées ici pour dater les concrétions sont détaillées dans Genty et al. (2004). Rappelons seulement qu'il faut entre 0,3 et $2 \mathrm{~g}$ de calcite pour l'U/Th TIMS (extrait à la microscie diamantée sur les sections polies) et de 20 à $50 \mathrm{mg}$ de calcite pour le ${ }^{14} \mathrm{C}$ AMS (extrait avec des forêts millimétriques). La précision analytique se situe entre $1 \%$ et $2 \%(2 \sigma)$ pour des échantillons "propres”, ce qui correspond à la plupart des cas ici. Par contre, elle est plus élevée pour des échantillons contaminés par des éléments détritiques qui apportent aussi du ${ }^{230} \mathrm{Th}$ et donc ont tendance à vieillir l'âge de l'échantillon (tabl. 1). Selon le diamètre des stalagmites (de 3 à $6 \mathrm{~cm}$ ici) et selon la vitesse de croissance $(0,1$ à $1 \mathrm{~mm} / \mathrm{an}$ en général ; voir Genty et Quinif, 1996 pour une revue de la variabilité des vitesses de croissance dans des stalagmites d'Europe de l'Ouest), le bloc de calcite extrait (de 0,5 à $1 \mathrm{~cm}$ de haut) couvrira entre 5 et 100 ans, durée généralement inférieure à l'incertitude analytique. Par contre, pour le cas de certains planchers et pour les voiles de calcite pariétaux où la lame d'eau est très fine, ou dans le cas de périodes froides peu propices à la croissance, la durée d'un bloc de plusieurs millimètres de haut peut représenter plusieurs siècles. En conséquence, dans le cas de très faibles croissances, l'interprétation des âges devra être prudente.

\section{RÉSULTATS ET DISCUSSIONS}

\section{Chronologie des dépôts stalagmitiques}

Les vingt-neuf analyses U/Th TIMS effectuées sur ce premier ensemble de concrétions donnent des informations sur l'âge minimum de l'éboulis d'entrée, l'âge de l'effondrement de la salle Hillaire et l'âge minimum des charbons de bois piégés à la base des concrétions.

\section{Âge de l'éboulis d'entrée}

Deux stalagmites ont été prélevées sur l'éboulis d'entrée : Chau-stm7 au sommet et Chau-stm8 à la base (photos 2 à 5). L'âge des premières phases de croissance des stalagmites donne l'âge minimum de la partie de l'éboulis qu'ils recouvrent. Ainsi, le haut de l'éboulis est plus ancien que $5800 \pm 90$ ans et la partie inférieure de l'éboulis est plus ancienne que 11500 \pm 170 ans.

Les éboulis colmatant les entrées de grottes sont nombreux et plusieurs hypothèses ont été émises pour les expliquer (Blanc et al., 1985; Escalon de Fonton, 1971): prédisposition tectonique et géomorphologique, variations climatiques, conjonction des deux facteurs précédents. Outre sa cause, il est important de connaître la dynamique de sa mise en place : éboulement brutal ou formation polyphasée? Les caractères géomorphologiques (taille des blocs, emplacement) auraient tendance à favoriser l'hypothèse d'un effondrement monophasé (Delannoy et al., 2003). Même si

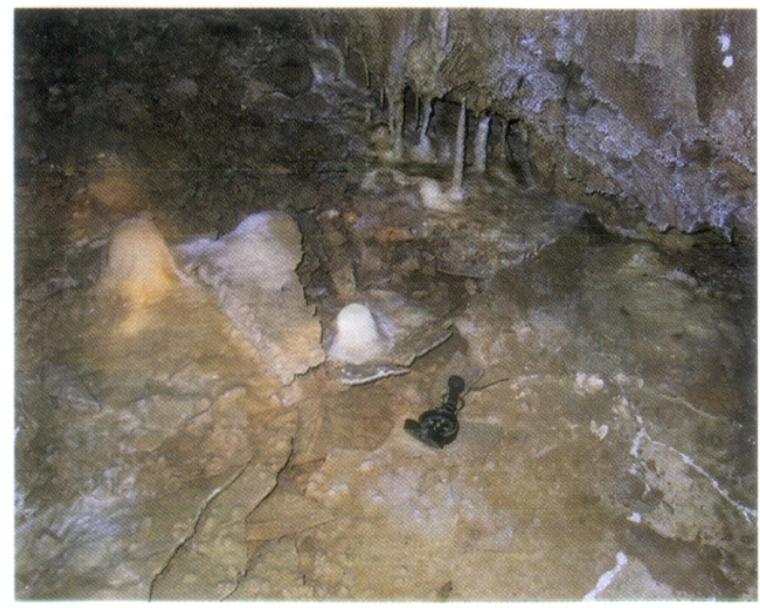

Photo 2 - Stalagmite Chau-stm 7 en place au sommet de l'éboulis d'entrée. 


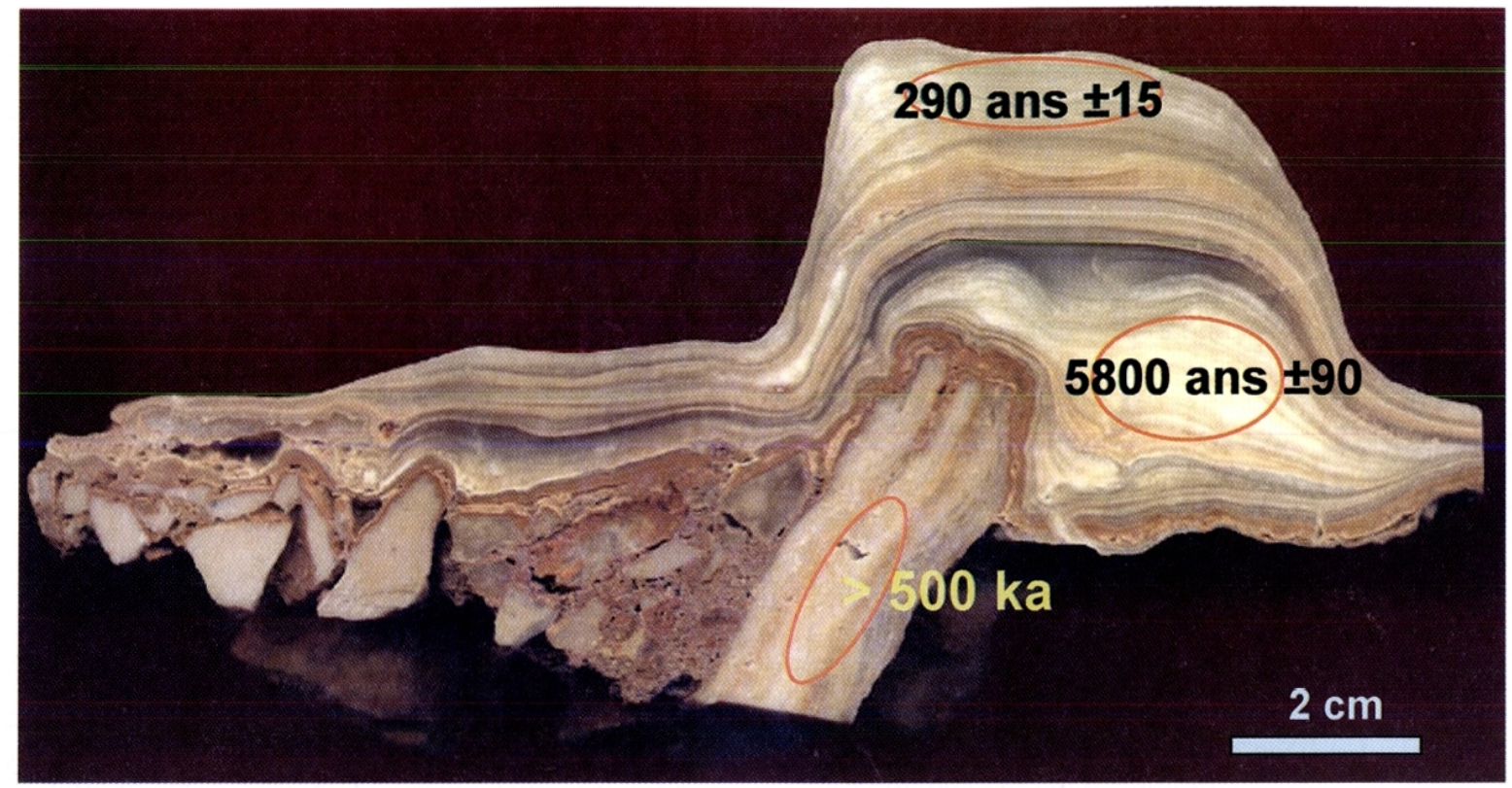

Photo 3 - Section polie verticale de la stalagmite Chau-stm 7 avec les âges U/Th. On note que les fragments constitutifs de l'éboulis, à la base de la stalagmite, sont parfois constitués de calcite (anciens remplissages) dont l'âge n'est pas mesurable ( $>500 \mathrm{ka})$. Les cercles orange correspondent aux échantillons prélevés.

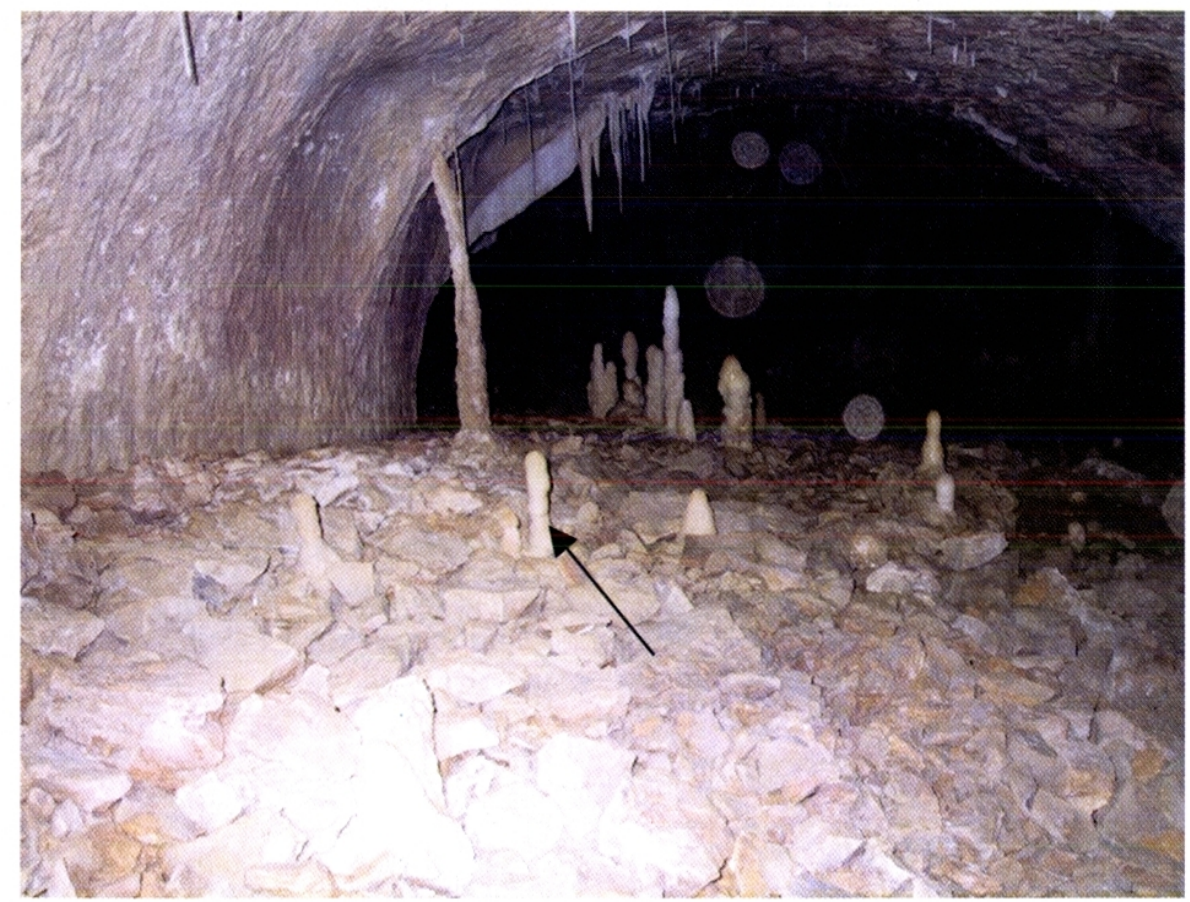

Photo 4 - Stalagmite Chau-stm8 en place vers la base de l'éboulis d'entrée.

les résultats issus de l'analyse de ces deux stalagmites semblent montrer que l'âge du sommet est plus récent que la base, il faudra analyser d'autres échantillons, à différentes positions sur l'éboulis, pour avoir une idée plus précise de la dynamique de sa mise en place. Il en est de même pour l'âge minimum de l'événement : il est certain que la partie inférieure est plus ancienne que 11500 ans, mais il existe d'autres stalagmites situées un peu plus bas sur l'éboulis qui pourraient éventuellement donner des âges encore plus anciens (travaux en cours). Il faut aussi garder à l'esprit que les stalagmites ne poussent pas lorsque le climat est trop froid et sec, il peut donc exister un hiatus de plusieurs milliers d'années entre la formation de l'éboulis et l'âge du premier dépôt de calcite qui commence à le recouvrir. Enfin, l'hypothèse d'un éboulement plus ancien est favorisée par les âges ${ }^{14} \mathrm{C}$ obtenus, d'une part sur les charbons et les peintures, et d'autre part, sur les ossements d'animaux trouvés dans la grotte. Ceux-ci montrent qu'il n'y a pas eu 


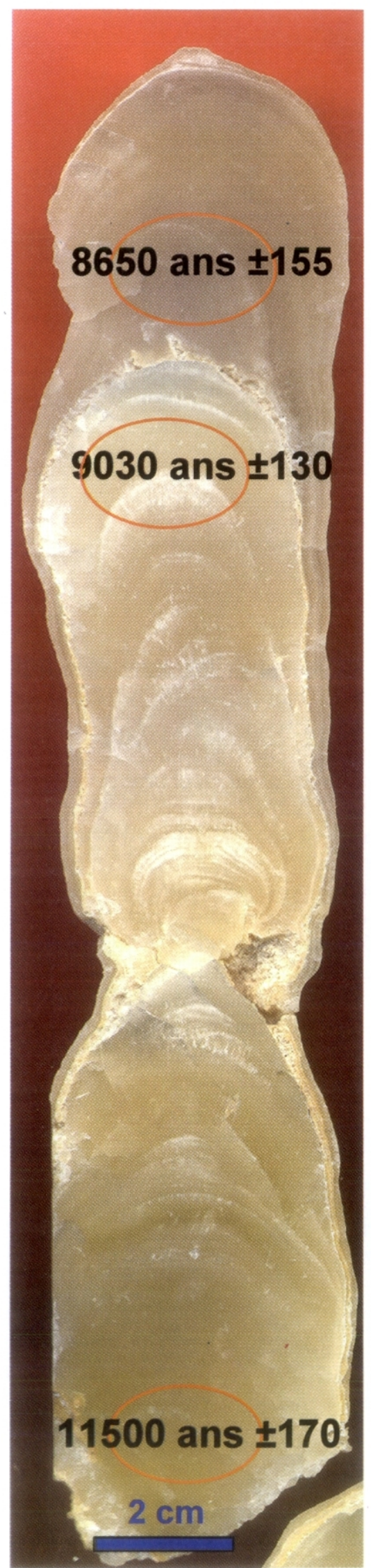

Photo 5 - Section polie verticale de la stalagmite ('hau-stm8 avec les âges U/Th. Les cercles orange correspondent aux échantillons prélevés. d'activité humaine postérieure à 24 ka BP (Valladas et al., 2001) et qu'aucun animal de grosse taille n'est entré dans la grotte après $22 \mathrm{ka}$ BP (Bocherens et al., 2003).

\section{Âge de l'effondrement de la salle Hillaire}

Il y a dans la salle Hillaire un effondrement circulaire du plancher stalagmitique d'environ dix mètres de diamètre qui met en évidence une coupe remarquable du sol archéologique sur une hauteur de plus de deux mètres. C'est l'un des rares endroits de la grotte où il est possible d'étudier le remplissage détritique et le sol archéologique en profondeur (Ferrier et al., 2003; Kervazo et al., 2003). Quatre datations U/Th ont été effectuées sur deux morceaux de ce plancher stalagmitique : Chau-pl $1(4,5 \mathrm{~cm}$ de haut $)$ et Chau-pl $2(2 \mathrm{~cm}$ de haut) (photos 6 à 10). Bien qu'ayant des incertitudes relativement élevées à cause des fortes teneurs en éléments détritiques (tabl. 1 et voir Genty et al., 2004 pour les détails), les âges obtenus donnent les informations chronologiques suivantes:

- le sol archéologique, avec les nombreux charbons qu'il contient en surface, a été recouvert par la calcite à la fin du stade isotopique 3, aux environs de 27800 \pm 1900 ans (tabl. 1);

- l'effondrement lui-même est postérieur à 7700 \pm 1630 ans, date la plus récente obtenue dans les couches supérieures de ces planchers;

- le hiatus bien visible sur la section verticale du plancher Chau-pll est très probablement dû aux conditions climatiques sévères du pléniglaciaire.

\section{Comparaison âges ${ }^{14} \mathrm{C}$ AMS des charbons} et âges U/Th TIMS des concrétions stalagmitiques C'est l'analyse au radiocarbone par accélération $\left({ }^{14} \mathrm{C}\right.$ AMS) effectuée sur des charbons trouvés au sol et sur quelques peintures directement qui a révélé l'ancienneté de l'art pariétal de la grotte Chauvet (Clottes et al., 1995; Valladas et al., 2001). Les deux ensembles de dates ainsi obtenus donnent des âges ${ }^{14} \mathrm{C}$ de 25 $27 \mathrm{ka} \mathrm{BP}$ et de 30-33 ka BP, tous en dehors des courbes de calibration $d{ }^{14} \mathrm{C}$ actuelles. En conséquence, ceuxci ne sont pas calibrés et leur âge "vrai" est probablement supérieur de 3 à 5 millénaires (Bard et al., 2004). Par contre, les âges U/Th obtenus sur la calcite sont

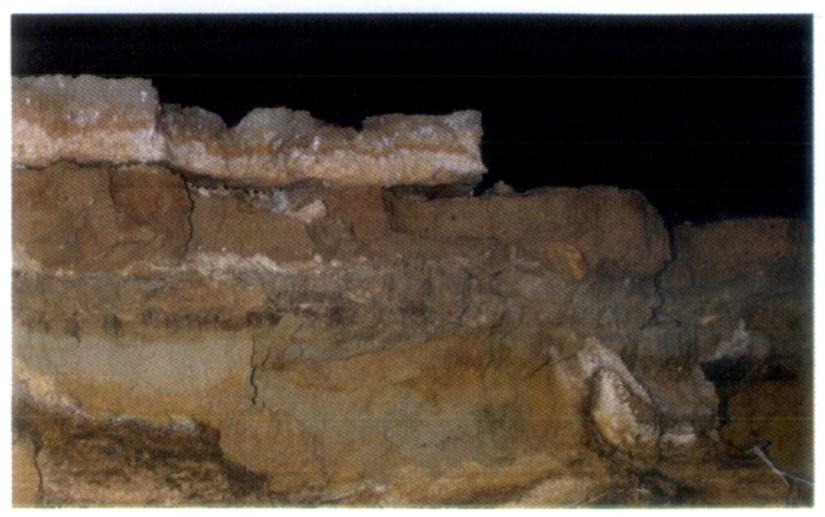

Photo 6 - Bordure de l'effondrement de la salle Hillaire montrant le plancher stalagmitique Chau-pll qui scelle le remplissage détritique. 


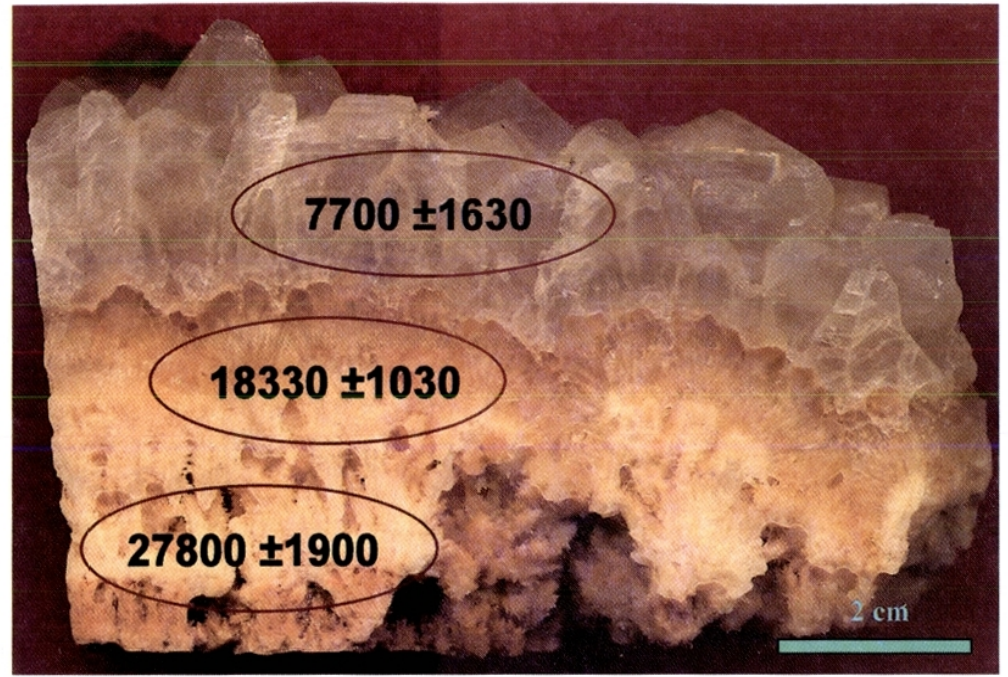

Photo 7 - Section polie verticale du plancher stalagmitique Chau-pll avec les âges U/Th. On note la discontinuité marquant la séparation entre une calcite claire et poreuse et une calcite plus compacte surmontée de scalénoèdres ; cette discontinuité correspond à l'arrêt de croissance lié au conditions climatiques extrêmes du dernier pléniglaciaire.

exprimés en années par rapport au présent, il faut donc tenir compte de ces différences lors de la comparaison des deux méthodes.

Sur l'ensemble des concrétions étudiées, quatre d'entre-elles recouvraient des charbons de bois : Chau-stm2 au niveau du seuil, Chau-stm3 dans la galerie des Mégacéros, Chau-pl1 et Chau-pl2 autour de l'effondrement de la salle Hillaire (tabl. 2). Un autre charbon (GC0022, tabl. 2) peut être considéré comme se situant, stratigraphiquement, sous une stalagmite puisqu'il a été trouvé à moins de deux mètres de l'emplacement de la stalagmite Chau-stm6, à la surface même du sol où celle-ci a poussé. Parmi ces cinq occurrences, il est important de noter que deux d'entre-elles permettent de confirmer, sans aucune ambiguïté, l'ancienneté des charbons : la base du plancher Chau-pl1, datée à $27800 \pm 1900$ ans, recouvre un charbon daté à $30020 \pm 350$ ans $\mathrm{BP}$; la base de la stalagmite Chau-stm6, datée à $32870 \pm 625$ ans, recouvre le sol où un charbon a été daté à 30550 \pm 370 ans BP (tabl. 2). On voit bien, pour ce dernier cxemple, que l'âge ${ }^{14} \mathrm{C}$ non calibré du charbon est légèrement inférieur à l'âge $U / T h$; y ajouter 3 millénaires comme pourrait le faire une calibration le rend cohérent avec la succession stratigraphique. Les autres cas montrent que, pendant 7 à 15 millénaires, aucun dépôt n'est venu recouvrir les charbons de bois laissés par les hommes préhistoriques, probablement à cause des conditions climatiques peu favorables au développement des concrétions.

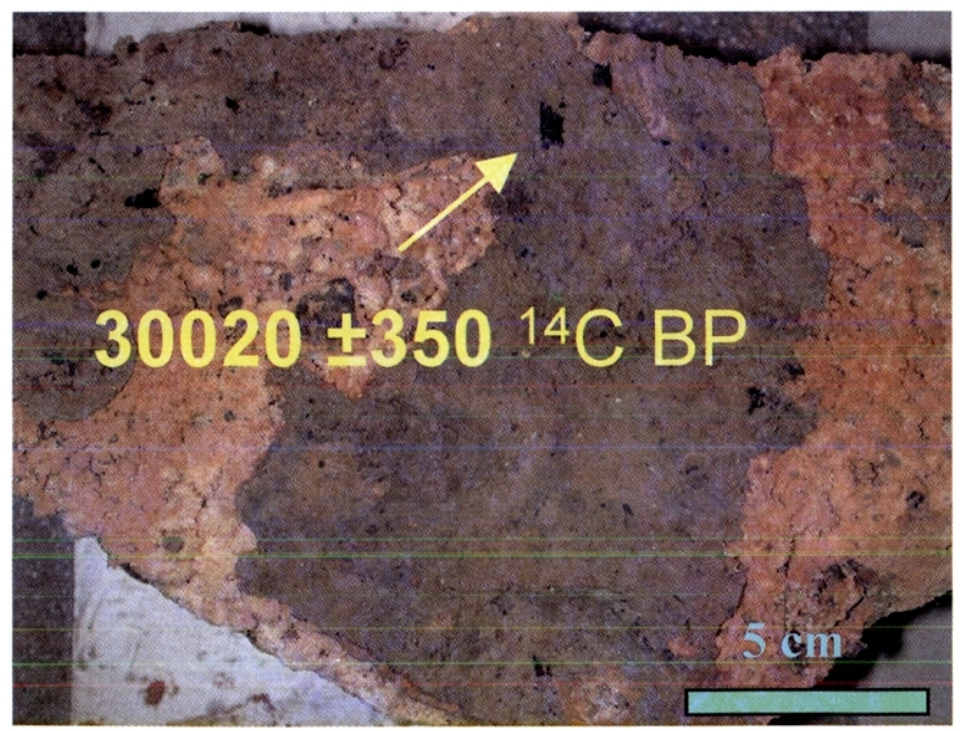

Photo 8 - Face inférieure du plancher stalagmitique Chau-pll mettant en évidence les charbons de bois recouverts par ce dernier. 


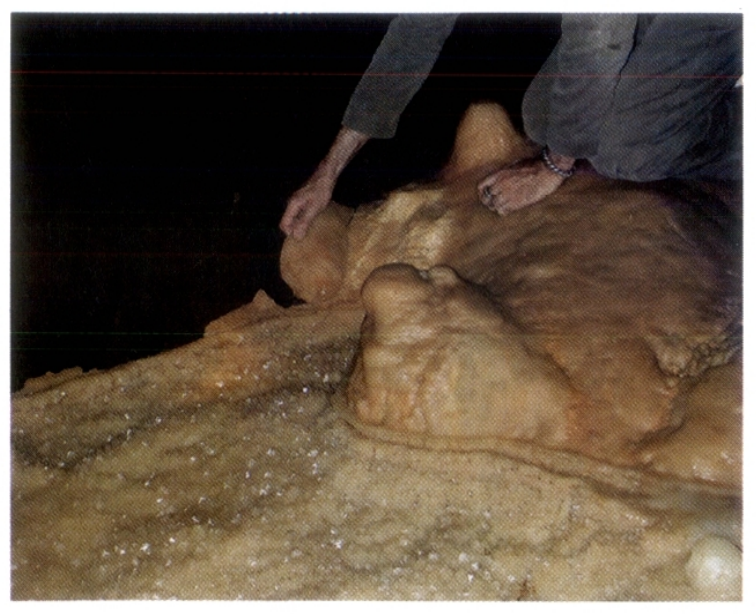

Photo 9 - Prélèvement du morceau de plancher stalagmitique Chau-pl2 au bord de l'effondrement de la salle Hilaire.

\section{Reconstitutions paléoenvironnementales par les isotopes stables de la calcite}

Nous présentons ici un état d'avancement de l'étude isotopique des concrétions de la grotte Chauvet, d'autres résultats viendront compléter ces premières courbes isotopiques par la suite, en particulier pour la période entre $11 \mathrm{ka}$ et $5 \mathrm{ka}$ où les analyses ne sont pas terminées mais où il existe des données (fig. 3). Les profils d'isotopes stables ont été effectués sur quatre stalagmites datées par U/Th ou ${ }^{14} \mathrm{C}$ (Chau-stm1, Chaustm3, Chau-stm4 et Chau-stm6) et recouvrent les derniers $34 \mathrm{ka}$. Le hiatus observé entre $\sim 24$ ka et $\sim 16 \mathrm{ka}$ sur la stalagmite Chau-stm6 correspond aussi à une période où aucune stalagmite ne semble avoir poussé dans la grotte Chauvet, très vraisemblablement à cause des conditions climatiques sévères du dernier maximum glaciaire (fig. 3). Ainsi, nos échantillons montrent deux périodes favorables à la croissance des stalagmites : la première avant $24 \mathrm{ka}$, où la stalagmite Chaustm6 a commencé à se développer avec une vitesse de croissance faible $(<0,02 \mathrm{~mm} / \mathrm{an})$; la seconde, après $16 \mathrm{ka}$, où la plupart des concrétions étudiées ont poussé avec des vitesses de croissance plus élevées (jusqu'à $0,68 \mathrm{~mm} / \mathrm{an}$ ).

Les isotopes stables de la calcite $\left(\delta^{18} \mathrm{O}\right.$ et $\left.\delta^{13} \mathrm{C}\right)$ montrent, eux aussi, une différence marquée entre la première phase de croissance de la fin du stade isotopique MIS 3 et l'Holocène (fig. 4 et 5 ) : le $\delta^{18} \mathrm{O}$ passe de $\sim 4 \%$ o autour de $30 \mathrm{ka} \grave{a} \sim-7 \%$ à $5 \mathrm{ka}$ et le $\delta^{13} \mathrm{C}$ passe de $\sim-4 \%$ (en moyenne) autour de $30 \mathrm{ka}$ à - $11 \%$ à $5 \mathrm{ka}$. Ils ont donc clairement enregistré la déglaciation et les différents événements qui l'ont marquée.

\section{Interprétation des signaux isotopiques}

Il est utile et recommandé, avant d'interpréter les signaux isotopiques d'une stalagmite, de voir si le dépôt s'est fait à l'équilibre isotopique ou non, car l'interprétation de ces signaux isotopiques ne serait alors pas directement liée aux variations climatiques. Il existe au moins deux critères qui permettent de contrôler cet équilibre : 1) vérifier que le signal isotopique ne s'enrichit pas vers les bords de la stalagmite, le long d'une même lamine; 2) vérifier qu'il n'y a pas de corrélation forte entre les valeurs de $\delta^{18} \mathrm{O}$ et de $\delta^{13} \mathrm{C}$ (Hendy, 1971). Dans le cas des stalagmites de la grotte Chauvet, la première condition est satisfaisante, par contre la seconde ne l'est pas pour les stalagmites Chau-stm6 et Chau-stm3. L'interprétation paléoclimatique faite ici n'est donc pas quantitative, même si la cohérence de l'enregistrement climatique pourrait nous y amener

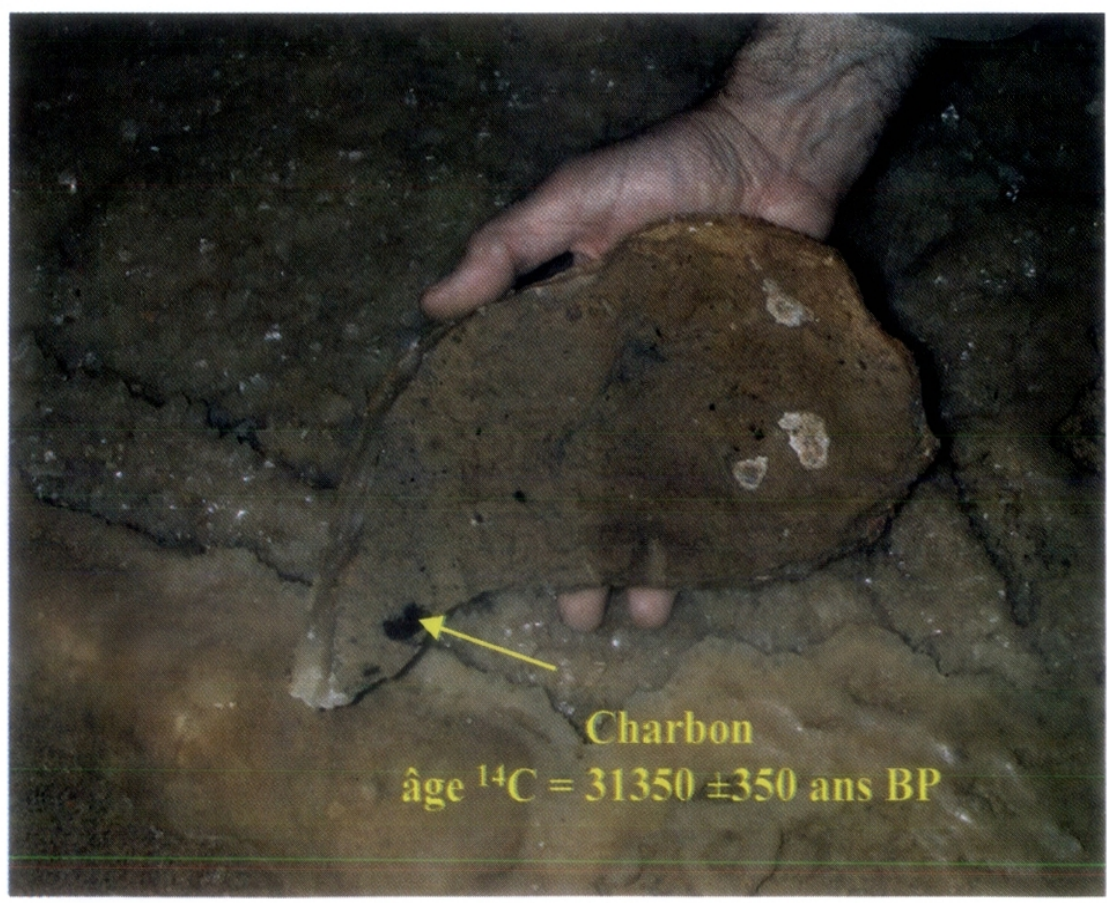

Photo 10 - Morceaux de charbons de bois piégés sous le plancher Chau-pl2. 


\begin{tabular}{|c|c|c|c|c|c|c|c|c|c|c|c|c|c|c|c|c|}
\hline Nom échantillon & $\begin{array}{l}\text { Hauteur totale } \\
\text { éch., } \mathrm{cm}\end{array}$ & $\begin{array}{l}\text { Position } \\
\mathrm{mm} / \text { base }\end{array}$ & \pm & $\begin{array}{l}{ }^{238} \mathrm{U} \\
\mathrm{ppm}\end{array}$ & \pm & $\begin{array}{l}{ }^{234} \mathrm{U} /{ }^{238} \mathrm{U} \\
\text { activité }\end{array}$ & \pm & $\begin{array}{c}{ }^{230} \mathrm{Th} /{ }^{234} \mathrm{U} \\
\text { activité }\end{array}$ & \pm & $\begin{array}{c}{ }^{230} \mathrm{Th} /{ }^{232} \mathrm{Th} \\
\text { activité }\end{array}$ & \pm & $\begin{array}{l}\text { Âge } A_{0} \\
\text { ans }\end{array}$ & "+" & "-" & $\begin{array}{c}\text { Âge U/Th corrigé } \\
\text { ans }\end{array}$ & $\begin{array}{c}\text { Erreur totale } \\
\text { ans }\end{array}$ \\
\hline Chau-stm1-U/Th-A & 35,7 & 266,5 & 3,5 & 0,22631 & 0,00014 & 1,01499 & 0,00260 & 0,03878 & 0,00080 & 6,1 & 0,1 & 4301 & 84 & 84 & 3601 & 391 \\
\hline Chau-stm1-U/Th-B & & 5 & 4,5 & 0,17211 & 0,00014 & 1,03373 & 0,00320 & 0,04281 & 0,00040 & 24,6 & 0,3 & 4757 & 39 & 39 & 4557 & 103 \\
\hline Chau-stm2-U/Th-B & 15,2 & 146,75 & 5,7 & 0,21349 & 0,00031 & 0,97310 & 0.0091 & 0,08176 & 0.0024 & 35,5 & 1,0 & 9280 & 224 & 222 & 9030 & 223 \\
\hline Chau-stm2-U/Th-E & & 54 & 4 & 0,18310 & 0,00015 & 1,03924 & 0.0031 & 0,11423 & 0.0008 & 511,4 & 6,9 & 13182 & 78 & 78 & 13112 & 78 \\
\hline Chau-stm2-U/Th-D & & 37 & 3 & 0,26954 & 0,00011 & 1,02821 & 0.0017 & 0,11768 & 0.0007 & 1080,8 & 7,2 & 13608 & 68 & 68 & 13558 & 68 \\
\hline Chau-stm2-U/Th-Ab & & 4 & 4 & 0,21473 & 0,00011 & 1,02786 & 0.0028 & 0,12141 & 0.0010 & 414,2 & 4,7 & 14068 & 95 & 95 & 13995 & 95 \\
\hline Chau-stm3-U/Th-E & 14,3 & 121,5 & 3,5 & 0,13684 & 0,00015 & 0,99359 & 0,00320 & 0,11503 & 0,00080 & 842,8 & 10,1 & 13291 & 71 & 71 & 13231 & 71 \\
\hline Chau-stm3-U/Th-D & & 66 & 3 & 0,13243 & 0,00011 & 0,98935 & 0,00460 & 0,12359 & 0,00100 & 623,4 & 5,8 & 14350 & 94 & 93 & 14290 & 94 \\
\hline Chau-stm3-U/Th-A2 & & 13 & 6 & 0,22586 & 0,00009 & 0,97411 & 0,00230 & 0,12680 & 0,00090 & 799,4 & 8,3 & 14755 & 84 & 84 & 14695 & 84 \\
\hline Chau-stm7-U/Th-C & 6 & 50 & 10 & 0,50874 & 0,00389 & 0,98178 & 0,00688 & 0,00285 & 0,00013 & 21,0 & 1,0 & 305 & 11 & 11 & 290 & 13 \\
\hline Chau-stm7-U/Th-B & & 10 & 10 & 0,97807 & 0,07690 & 0,98536 & 0,00883 & 0,05189 & 0,00084 & 119,6 & 2,1 & 5797 & 92 & 92 & 5797 & 92 \\
\hline Chau-stm7-U/Th-A & & -10 & & 0,39578 & 0,00240 & 1,01247 & 0,00686 & 1,00065 & 0,01025 & 92,6 & 1,1 & $>500000$ & ind. & ind. & $>500000$ & ind. \\
\hline Chau-stm8-U/Th-C & 22,2 & 192,5 & 12,5 & 0,33660 & 0,00272 & 0,95901 & 0,01180 & 0,07637 & 0,00131 & 1676,1 & 27,3 & 8648 & 155 & 154 & 8648 & 155 \\
\hline Chau-stm8-U/Th-B & & 162,5 & 12,5 & 0,34519 & 0,00264 & 0,95640 & 0,00947 & 0,07958 & 0,00110 & 715,2 & 10,3 & 9026 & 132 & 131 & 9026 & 132 \\
\hline Chau-stm8-U/Th-A & & 12,5 & 12,5 & 0,21166 & 0,00152 & 0,91714 & 0,00716 & 0,10016 & 0,00135 & 212,4 & 3,2 & 11500 & 172 & 171 & 11500 & 172 \\
\hline Chau-stm5-U/Th-A & 28 & 7,5 & 7,5 & 0,31270 & 0,00170 & 0,97772 & 0,00506 & 0,11190 & 0,00301 & 7,1 & 0,2 & 12912 & 370 & 368 & 11175 & 1163 \\
\hline Chau-stm6-U/Th-F & & 664 & 8 & 0,28477 & 0,00157 & 0,57000 & 0,00490 & 0,09910 & 0,00120 & 210,7 & 2,6 & 11498 & 152 & 152 & 11498 & 152 \\
\hline Chau-stm6-U/Th-E & & 511 & 7 & 0,32860 & 0,00175 & 0,55440 & 0,00460 & 0,10590 & 0,00180 & 1119,0 & 19,0 & 12356 & 230 & 229 & 12356 & 230 \\
\hline Chau-stm6-U/Th-D & & 374,5 & 7,5 & 0,31018 & 0,00173 & 0,55920 & 0,00470 & 0,11490 & 0,00210 & 976,8 & 17,8 & 13488 & 272 & 270 & 13488 & 271 \\
\hline Chau-stm6-U/Th-J & & 324,5 & 5,5 & 0,34780 & 0,00221 & 0,53510 & 0,00740 & 0,11810 & 0,00190 & 644,0 & 8,3 & 13920 & 252 & 250 & 13920 & 251 \\
\hline Chau-stm6-U/Th-B & & 215,5 & 7,5 & 0,25651 & 0,00147 & 0,58160 & 0,00730 & 0,12370 & 0,00350 & 493,6 & 13,9 & 14591 & 458 & 454 & 14591 & 456 \\
\hline Chau-stm6-U/Th-I & & 152,5 & 7,5 & 0,32090 & 0,00176 & 0,55510 & 0,00650 & 0,12700 & 0,00190 & 625,8 & 7,7 & 15044 & 254 & 253 & 15044 & 254 \\
\hline Chau-stm6-U/Th-H & & 92 & 5 & 0,50650 & 0,00266 & 0,55576 & 0,00609 & 0,20954 & 0,00368 & 134,4 & 2,1 & 26446 & 574 & 567 & 26446 & 571 \\
\hline Chau-stm6-U/Th-A3 & & 37,5 & 2,5 & 0,44080 & 0,00188 & 0,54153 & 0,00440 & 0,22694 & 0,00638 & 182,4 & 5,2 & 29128 & 1011 & 996 & 29128 & 1004 \\
\hline Chau-stm6-U/Th-A2 & & 17,5 & 2,5 & 0,37720 & 0,00171 & 0,56455 & 0,00464 & 0,22627 & 0,00885 & 152,9 & 6,0 & 28882 & 1370 & 1344 & 28882 & 1357 \\
\hline Chau-stm6-U/Th-A & & 10 & 5 & 0,44810 & 0,00219 & 0,54449 & 0,00435 & 0,25093 & 0,00370 & 81,8 & 1,2 & 32872 & 625 & 618 & 32872 & 622 \\
\hline Chau-pl1-U/Th-C & 4,5 & 38 & 5 & 0,07700 & 0,00048 & 0,99619 & 0,00938 & 0,08687 & 0,00249 & 4,4 & 0,1 & 9887 & 300 & 299 & 7704 & 1633 \\
\hline Chau-pl1-U/Th-B & & 21 & 4 & 0,69800 & 0,00534 & 0,98590 & 0,00515 & 0,16618 & 0,00213 & 12,7 & 0,2 & 19776 & 278 & 277 & 18327 & 1028 \\
\hline Chau-pl1-U/Th-A & & 10 & 5 & 1,00964 & 0,00697 & 0,99964 & 0,00611 & 0,23932 & 0,00280 & 13,5 & 0,2 & 29747 & 410 & 408 & 27787 & 1915 \\
\hline Chau-pl2-U/Th-A & 2 & 10 & 5 & 0,14680 & 0,00088 & 1,00249 & 0,00720 & 0,14775 & 0,00247 & 11,4 & 0,2 & 17380 & 323 & 322 & 15951 & 1106 \\
\hline
\end{tabular}

\section{Tableau 1}

\begin{tabular}{|c|c|c|c|c|c|c|c|}
\hline Nom échantillon & $\mathrm{mm} /$ base & \pm & $\begin{array}{c}\text { Activité }{ }^{14} \mathrm{C} \\
\text { mesurée, pMC }\end{array}$ & $\pm(1 \sigma)$ & $\begin{array}{l}\text { Agge Conventionel, } \\
\text { ans BP }\end{array}$ & $\pm(1 \sigma)$ & $\begin{array}{c}\text { cal. }{ }^{14} \mathrm{C} \\
\text { âge/2000, }(2 \sigma)\end{array}$ \\
\hline Chau-stm1-14C-B & 355 & 0,4 & 79,75 & 0,55 & 1818 & 56 & $1000-100$ \\
\hline Chau-stm4-14C-A & 2 & 0,4 & 53,87 & 0,45 & 4969 & 67 & $6000-4600$ \\
\hline Chau-stm4-14C-B & 202 & 0,4 & 55,85 & 0,46 & 4679 & 66 & $5700-4200$ \\
\hline Chau-stm4-14C-C & 205 & 0,4 & 56,27 & 0,35 & 4619 & 50 & $5700-4200$ \\
\hline Chau-stm4-14C-Dter & 335 & 0,4 & 85,9 & 0,80 & 1221 & 75 & $1350-550$ \\
\hline Chau2-Charbon (GifA 102572) & -1 & 0,5 & 3,65 & 0,14 & 26590 & 300 & \\
\hline Chau3-Charbon & -1 & 0,5 & 6,61 & 0,11 & 21822 & 130 & \\
\hline Chau-pl1-Charbon (GifA 102573) & -1 & 0,5 & 2,38 & 0,11 & 30020 & 350 & \\
\hline GC0028 (GifA 101458) & niv. archéol. & & 1,63 & 0,12 & 33100 & 590 & \\
\hline Chau-pl2-Charbon (GifA 102574) & -1 & 0,5 & 2,02 & 0,11 & 31350 & 440 & \\
\hline GC0022 (GifA 102566) & niv. archéol. & & 2,23 & 0,10 & 30560 & 370 & \\
\hline
\end{tabular}




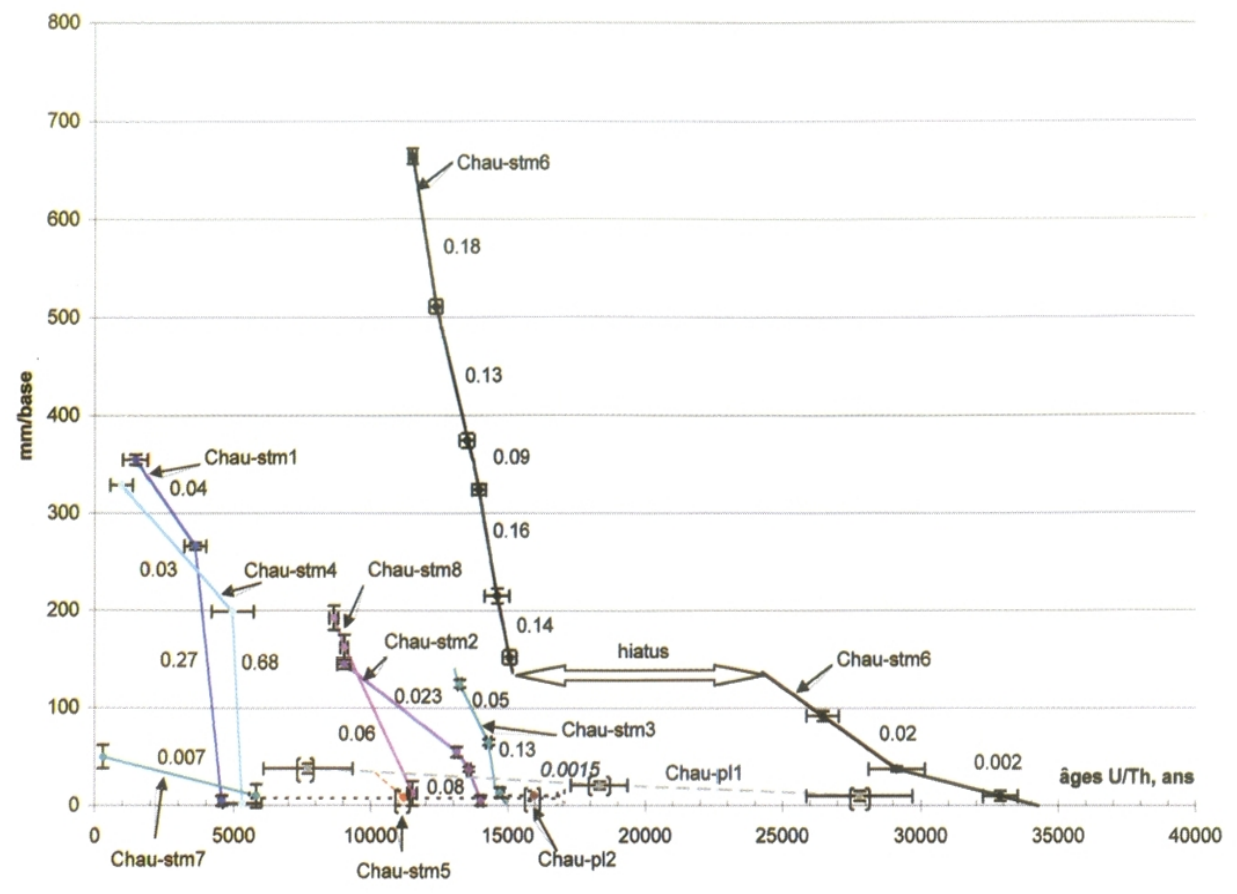

Fig. 3 - Vitesses de croissance des stalagmites et des planchers stalagmitiques de la grotte Chauvet. Les valeurs indiquées sur le graphique sont en mm/an. Il s'agit de vitesses moyennes calculées avec les âges U/Th. Les erreurs relatives sur les vitesses de croissance s'échelonnent entre $\pm 10 \%$ et $\pm 89 \%$, cette dernière valeur concernant le plancher Pll dont la croissance est très faible (in Genty et al., 2004).

(une estimation des paléotempératures est théoriquement possible avec le $\delta^{18} \mathrm{O}$ de la calcite et des hypothèses sur le $\delta^{18} \mathrm{O}$ de l'eau d'infiltration au moment du dépôt). Par contre, nous appuyons nos interprétations sur les études isotopiques effectuées sur l'eau d'infiltration et sur des dépôts de calcite modernes prélevés

Tabl. 1 (ci-contre) - (in Genty et al., 2004) - Résultats des analyses uranium-thorium. Une correction sur le ${ }^{230} \mathrm{Th}$ initial a été effectuée pour tous les échantillons dont le rapport ${ }^{230} \mathrm{Th} /{ }^{232} \mathrm{Th}$ est inférieur à 50 . Un rapport ${ }^{230} \mathrm{Th} /{ }^{232} \mathrm{Th}$ initial de 1 (activité) a été choisi avec une incertitude de $\pm 0,5$ qui est prise en compte dans l'erreur finale (colonne erreur totale), d'où les grandes marges d'incertitudes sur l'âge des échantillons contaminés par du thorium détritique. Le rapport d'activité ${ }^{234} \mathrm{U} /{ }^{238} \mathrm{U}$ inhabituel de l'échantillon Chau-stm6 $(\sim 0,55$ au lieu de $\sim 1$ en général) est très probablement dû à une eau d'infiltration déjà appauvrie en ${ }^{234} \mathrm{U}$ comme le démontre le rapport ${ }^{234} U /{ }^{238} U$ de $0,8838 \pm 0,0054$ mesuré sur de l'eau moderne prélevée à environ $5 \mathrm{~m}$ de la stalagmite. Ceci montre que, localement, la minéralisation des eaux s'effectue à travers des formations (sol/roche) lessivées et appauvries en ${ }^{234} \mathrm{U}$. La colonne hauteur représente la hauteur totale de la stalagmite ou du plancher stalagmitique en $\mathrm{cm}$. La stalagmite Chau-stm4 a une hauteur totale de $33,4 \mathrm{~cm}$.

Tabl. 2 (ci-contre) - (in Genty et al., 2004) - Résultats des analyses ${ }^{14} \mathrm{C}$ des charbons piégés sous les spéléothèmes et sur Chau-stm4 et le sommet de Chau-stm1. Les âges ${ }^{1+} \mathrm{C}$ des stalagmites ont été corrigés d'une proportion de carbone mort de $4 \%$ (par comparaison U/Th- ${ }^{14} \mathrm{C}$ ) et ensuite calibrés suivant les courbes de calibration de Stuiver et Kra. 1986). Les erreurs finales prennent en compte non seulement l'erreur analytique, mais aussi l'erreur sur la proportion de carbone mort ainsi que l'erreur de la courbe de calibration. Les charbons trouvés juste sous la base des spéléothèmes sont positionnés à - $1 \mathrm{~mm} / \mathrm{base}$. Le charbon GC0028 a été prélevé dans la salle Hillaire, à $5 \mathrm{~cm}$ sous le plancher stalagmitique (identique à Chau-pl! et Chau-p/2). Il appartient au même niveau archéologique que les charbons Chau-pll-charbon et Chau-pl2charbon. Le charbon GC0022 a été trouvé sur le sol de la galerie des Croisillons, à environ deux mètres de la stalagmite Chau-stm6 qui a poussé sur ce sol. dans la grotte Chauvet et dans d'autres sites depuis plusieurs années. Pour résumer, le $\delta^{18} \mathrm{O}$ de la calcite dépend de plusieurs facteurs qui parfois peuvent s'opposer : la température à laquelle la calcite a précipité $\left(-0,24 \%{ }^{\circ} \mathrm{C}\right.$; O'Neil et al., 1969; Kim et O'Neil, 1997); le $\delta^{18} \mathrm{O}$ de l'eau d'infiltration qui est proche de la moyenne annuelle du $\delta^{18} \mathrm{O}$ des eaux de pluie, luimême dépendant de la température de condensation dans le nuage. Si la relation qui lie le $\delta^{18} \mathrm{O}$ de la calcite et la température est bien définie (O'Neil et al., 1969; Kim et O'Neil, 1997), celle qui lie le $\delta^{18} \mathrm{O}$ des eaux de pluie avec la température extérieure n'est pas sûre ( $\mathrm{R}^{2}$ souvent faible) et a probablement changé au cours du temps. Enfin, le $\delta^{18} \mathrm{O}$ de la pluie est dépendant du $\delta^{18} \mathrm{O}$ de la source (océan), du chemin parcouru entre la source et le site, et de la quantité d'eau condensée durant le parcours. Selon le site, le $\delta^{18} \mathrm{O}$ de la calcite sera donc interprété comme lié à la pluviométrie (effet de masse, cas de la grotte Soreq en Israël; BarMatthews et al., 1996 et 1997) ou comme lié à la température extérieure (dont la moyenne annuelle est proche de la température de la grotte; cas d'une grotte des Alpes en Autriche; Spötl et al., 2003). En conséquence, à cause de la complexité de l'interprétation du $\delta^{18} \mathrm{O}$ et parce que nous comprenons mieux l'origine des variations du $\delta^{13} \mathrm{C}$, nous basons la reconstitution paléoclimatique des stalagmites de la grotte Chauvet essentiellement sur les isotopes du carbone.

Le $\delta^{13} \mathrm{C}$ est sensible à tous les changements qui affectent le sol car 80 à $90 \%$ du carbone des stalagmites provient du $\mathrm{CO}_{2}$ du sol, le reste provenant de la dissolution du calcaire encaissant (Genty et al., 1999). 

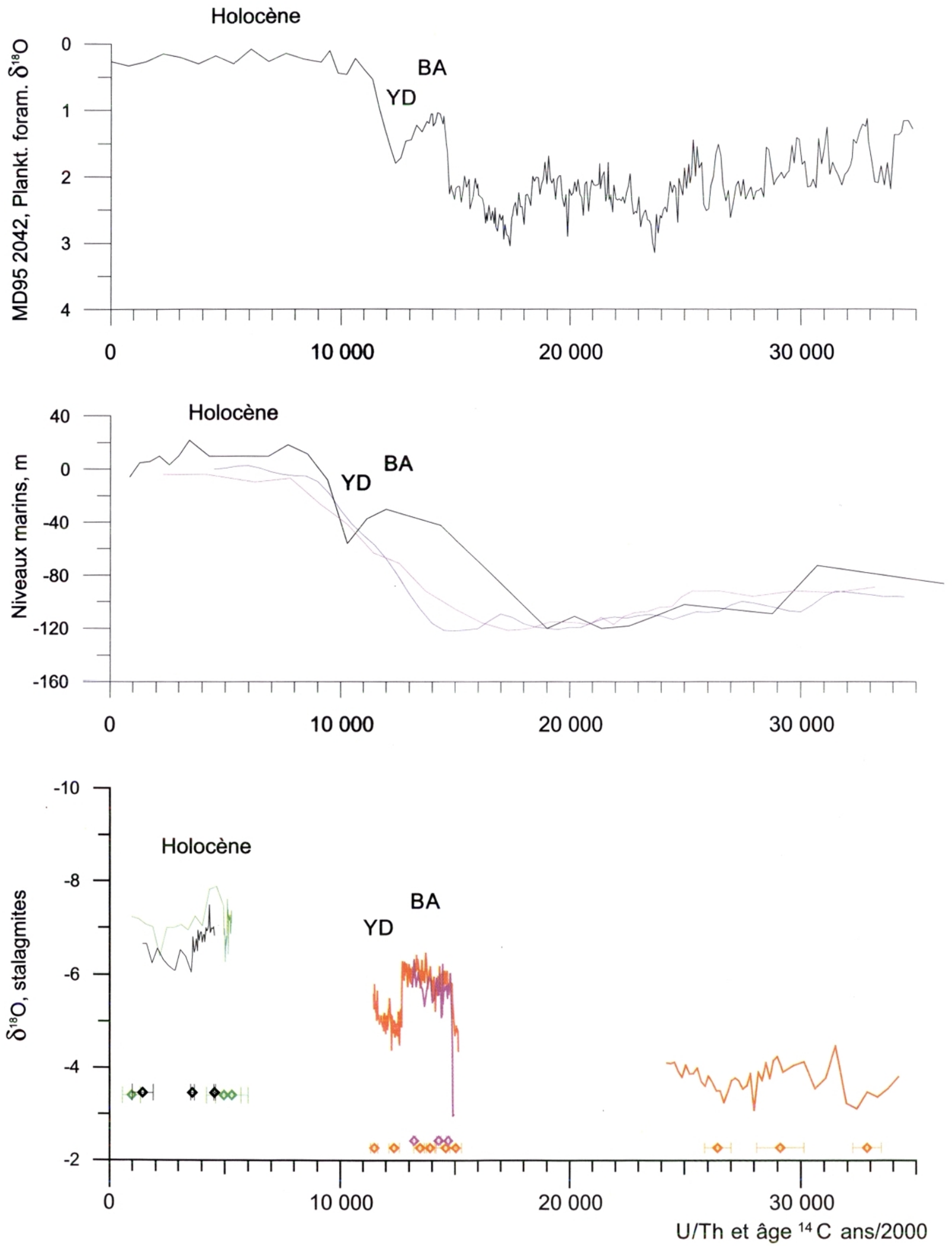

Fig. 4 - Profils en $\delta^{18} \mathrm{O}$ de la calcite des stalagmites de la grotte Chauvet (en bas). Les losanges représentent les points datés avec leur barre d'erreur à 2 o. Comparaison avec un enregistrement marin au large du Sud du Portugal (MD 95-2042; Shackelton et Hall, 2001) et les variations du niveau marin (SPECMAP : Martinson et al., 1987 (violet); Cortijo et al., 1995 (noir) et Waelbroeck et al. 1995 (bleu)). 

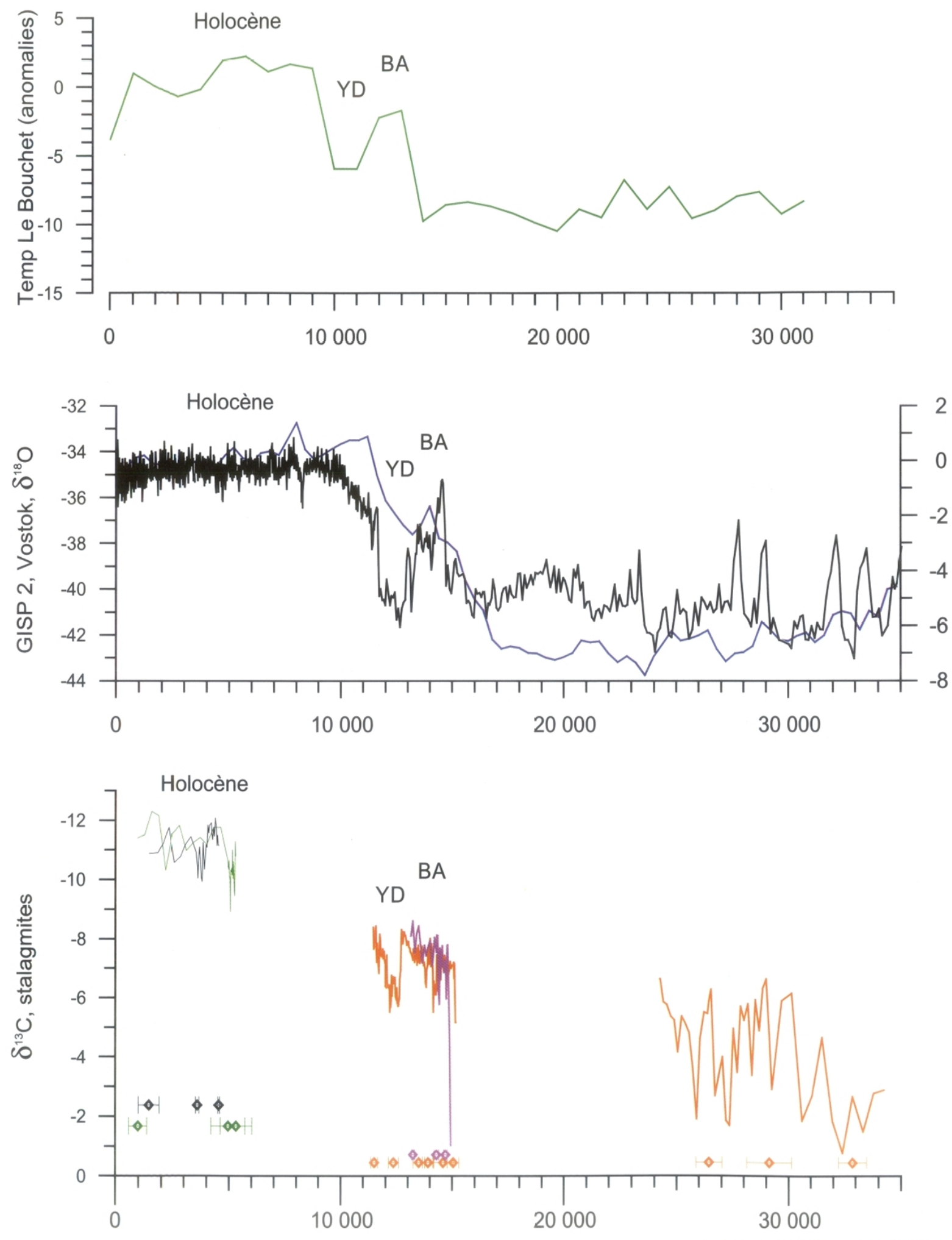

U/Th et ${ }^{14} \mathrm{C}$ âge, ans/2000

Fig. 5 - Profils en $\delta^{13} \mathrm{C}$ de la calcite des stalagmites de la grotte Chauvet (en bas). Les losanges représentent les points datés avec leur barre d'erreur à 2 o. Comparaison avec: 1) en haut, l'enregistrement pollinique du lac du Bouchet (Velay: de Beaulieu et Reille, 1992: Guiot et al., 1993) ; 2) au milieu. les enregistrements des carottes de glace (GISP2, Vostok (bleu): Grootes et al. . 1993: Cuffey et Vimeux, 2001). Les valeurs particulièrement élevées de la base de la stalagmite (hau-stm. 3 sont sans doute dues à des contaminations par des éléments détritiques, visibles sur les premiers millimètres de la section polie. 


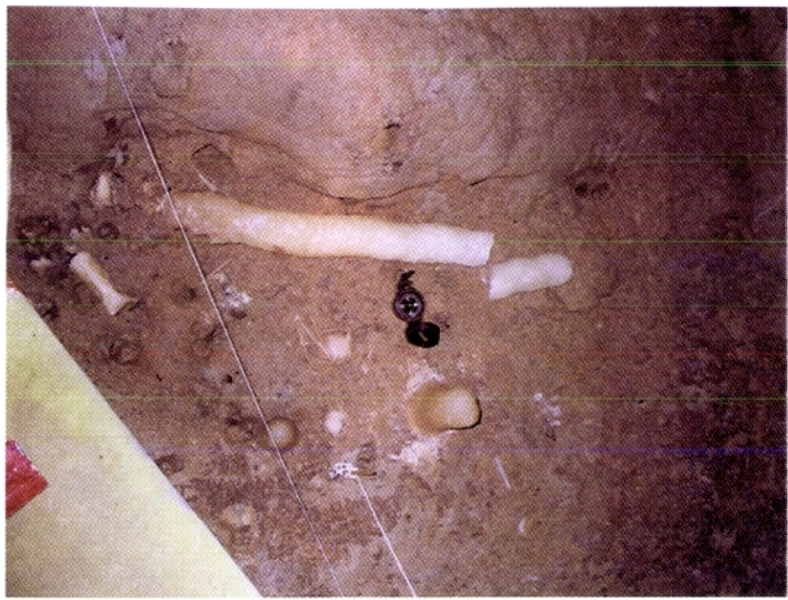

Photo 11 - Stalagmite Chau-stm6 trouvée cassée en bordure du cheminement.

Les variations du $\delta^{13} \mathrm{C}$ reflètent ainsi essentiellement les variations de densité du couvert végétal et de l'activité biopédologique du sol au-dessus de la grotte (la température joue aussi un rôle non négligeable sur le fractionnement isotopique; voir McDermott, 2004 et Genty et al., 2003 pour les discussions sur l'interprétation des isotopes stables dans les stalagmites). En période chaude et humide, la production de $\mathrm{CO}_{2}$ biogénique du sol est forte, sa valeur de $\delta^{13} \mathrm{C}$ est faible (entre - $20 \%$ et - $25 \%$ ), la calcite qui se forme alors à partir de l'eau d'infiltration qui a dissout ce $\mathrm{CO}_{2}$ aura un $\delta^{13} \mathrm{C}$ faible. Les valeurs du $\delta^{13} \mathrm{C}$ obtenues sur des stalactites actives de la grotte Chauvet sont comprises entre - $9 \%$ et - $11 \%$, valeurs proches de celles de l'Holocène et caractérisent donc un climat tempéré relativement chaud et humide (fig. 5). Par contre, en période plus froide, le $\delta^{13} \mathrm{C}$ des échantillons de Chauvet atteint des valeurs de $-4 \%$, voire $-1 \%$ autour de $33 \mathrm{ka}$ (fig. 5), conséquence probable d'une réduction importante de l'activité végétale et de la production de $\mathrm{CO}_{2}$ biogénique. La première période de croissance, autour de $30 \mathrm{ka}$, est caractérisée par une grande instabilité dans les valeurs du $\delta^{13} \mathrm{C}$ qui montrent des variations rapides de plus de $4 \%$ (fig. 5). Les enregistrements glaciaires (GISP2, fig. 5) et marins (MD 95-2042, fig. 4) montrent aussi un climat instable pour cette période avec notamment les derniers événements abrupts que sont les Dansgaard-Oeschger (DO) avant le pléniglaciaire. Entre $\sim 24250$ ans et $\sim 15160$ ans, la stalagmite Chau-stm6 s'est arrêtée de pousser (fig. 3). Ce hiatus, d'environ 9000 années, est à peine marqué dans la stalagmite par une couche brune et fine située à $13,65 \mathrm{~cm}$ de la base (photos 11 et 12 ) mais correspond certainement à une période froide et sèche car aucune autre stalagmite, parmi celles échantillonnées jusqu'ici, n'a poussé. Les autres enregistreurs climatiques montrent un niveau des océans minimal et les isotopes des enregistrements marins et glaciaires caractérisent des températures très faibles (fig. 4 et 5). Beaucoup plus proches de la grotte Chauvet, les enregistrements polliniques des lacs du Velay ont permis de reconstituer l'évolution de la température et de la pluviométrie pendant cette période (de Beaulieu et Reille, 1992; Guiot et al., 1993) : la température est plus basse de -8 à $-12^{\circ} \mathrm{C}$ que la température moyenne actuelle (fig. 5) et le déficit des précipitations moyennes annuelles atteint $700 \mathrm{~mm}$. C'est donc un climat froid et sec, peu favorable au développement des stalagmites.

L'amélioration climatique semble arriver brusquement : la transition vers le Bølling-Allerød est synchrone sur ces deux échantillons et a lieu dès 15160

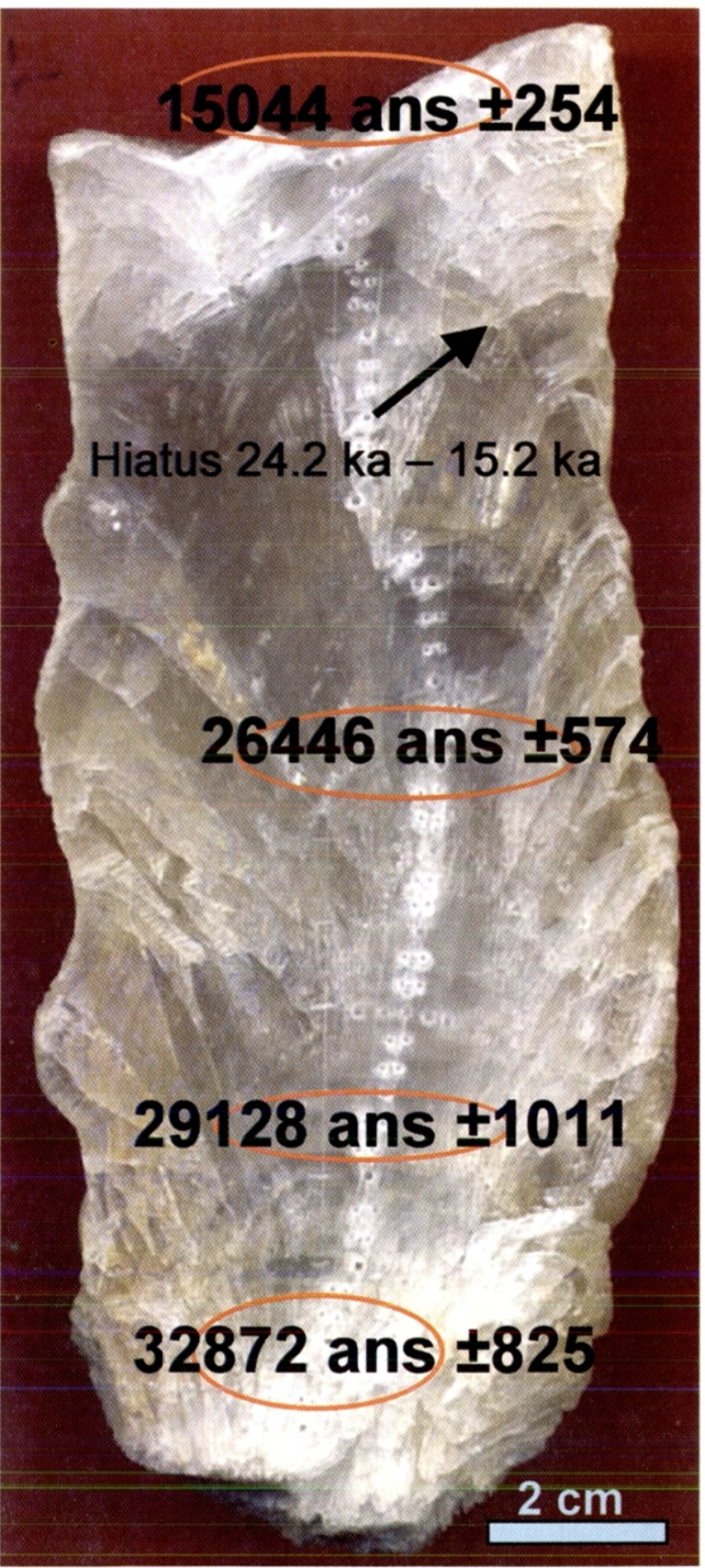

Photo 12 - Section polie verticale de la base de la stalagmite Chau-stm6 avec les âges $U / T h$. Les cercles orange correspondent aux échantillons prélevés. Le hiatus de croissance entre $24,2 \mathrm{ka}$ et 15,2 ka apparaît comme une fine couche brune. Les trous le long de l'axe de croissance correspondent aux échantillons prélevés pour les analyses d'isotopes stables. 


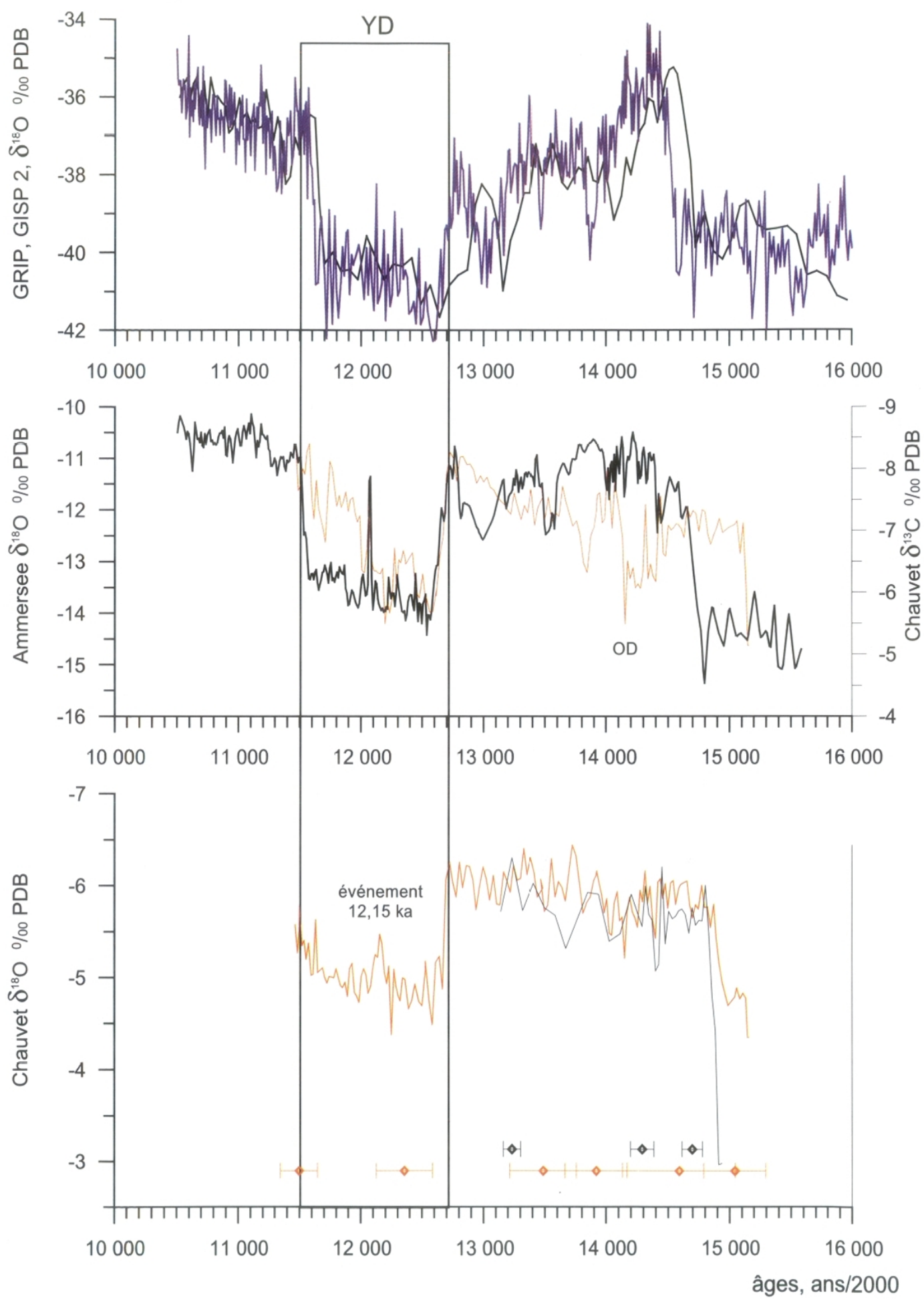

Fig. 6 - Enregistrement du Bølling-Allerød (BA) et du Younger-Dryas (YD) dans les isotopes stables de la stalagmite Chau-stm6 (en bas, en rouge : $\delta^{18} \mathrm{O}$; au milieu, en rouge : $\delta^{13} \mathrm{C}$ ) et dans ceux de la stalagmite Chau-stm3 (en bas, en noir). Comparaison avec : 1) en haut, les enregistrements glaciaires du Groenland (GISP2 en bleu; GRIP en noir; Dansgaard et al., 1993; Grootes et al., 1993); 2) au milieu (en noir) l'enregistrement du lac Ammersee (Sud de l'Allemagne; von Grafenstein et al., 1999). On note la similitude de ces enregistrements avec la transition rapide vers le BA et surtout la brusque transition vers le YD entre 12.7 et $12.6 \mathrm{ka}$. Un petit événement climatique (chaud?) est visible à 12.15 ka sur la stalagmite Chau-stm6 et sur l'enregistrement d'Ammersee. OD : older Dryas.

\pm 250 ans pour Chau-stm6 et dès $14960 \pm 90$ ans pour Chau-stm3, en accord avec les âges des autres archives (fig. 5). En moins de 150 ans, la valeur du $\delta^{13} \mathrm{C}$ chute de plus de $2 \%$ pour Chau-stm6 et de plus de $7 \%$ pour
Chau-stm.3 (pour ce dernier échantillon, une contamination par des particules détritiques est probable dans les premiers millimètres de sa base et pourrait expliquer les valeurs extrêmement élevées). La stalagmite 
Chau-stm6 a remarquablement bien enregistré le BA et le YD avec une vitesse de croissance relativement élevée (de 0,1 à $0,2 \mathrm{~mm} / \mathrm{an}$; fig. 3 et 6). Les isotopes de la calcite de Chau-stm6 et Chau-stm 3 montrent une amélioration climatique au sein même du BA, tendance inverse de celle observée dans les carottes de glace du Groenland (fig. 6). Cette tendance est aussi observée dans l'un des rares enregistrements continentaux à haute résolution d'Europe : le lac Ammersee au Sud de l'Allemagne (von Grafenstein et al., 1999). Il semblerait donc qu'il existe des différences régionales importantes pour cette période, et il est possible que l'enregistrement glaciaire du Groenland soit fortement influencé par la dynamique de fonte de la calotte fennoscandienne. On note aussi que, pendant le BA, le profil isotopique en $\delta^{13} \mathrm{C}$ de Chau-stm6 indique deux refroidissements : le premier, le plus intense, à 14150 ans \pm 300 et le second à 13815 ans \pm 250 (fig. 6).

Mais l'événement le plus marquant est sans doute l'arrivée du Younger-Dryas, il y a 12700 ans $( \pm 250$ ans) : le $\delta^{13} \mathrm{C}$ augmente brusquement de plus de $2 \%$ en moins de 80 ans (interpolation linéaire entre les points datés) témoignant d'une brusque diminution de la production de $\mathrm{CO}_{2}$ biogénique et d'une baisse de température (fig. 6). En même temps, les valeurs de $\delta^{18} \mathrm{O}$ augmentent de plus de $1,5 \%$, ce qui est pourrait signifier une baisse de température de plus de $6^{\circ} \mathrm{C}$ si l'on considère le fractionnement à l'équilibre et aucun changement dans la valeur du $\delta^{18} \mathrm{O}$ des eaux de pluie (fig. 6). Ce refroidissement abrupt coïncide parfaitement avec celui enregistré à GISP2, GRIP, au lac Ammersee et dans les lacs du Velay; il correspond à une baisse des températures moyennes comprise entre 5 et $10^{\circ} \mathrm{C}$ (fig. 6). Si la baisse de la température ne fait pas de doute, une diminution de la pluviométrie ne paraît pas évidente car la vitesse de croissance de Chau-stm6 n'a quasiment pas changé entre le BA et le YD $(0,18 \mathrm{~mm} / \mathrm{an}$; fig. 3$)$. Le YD apparaît à Chauvet comme froid mais toujours humide (?).

Alors que le $\delta^{13} \mathrm{C}$ semble indiquer une amélioration climatique lente mais régulière dès le début du YD, juste après le refroidissement brutal, le profil en $\delta^{18} \mathrm{O}$ montre l'existence d'un petit événement il y a 12150 ans \pm 230 (fig. 6). Celui-ci va dans le sens d'un réchauffement et coïncide avec un événement similaire enregistré dans le lac Ammersee (fig. 6). Le fait qu'il soit très peu marqué dans le $\delta^{13} \mathrm{C}$ est peut-être dû à la rapidité de l'événement et à l'inertie du système biopédologique.

L'absence de donnée entre la fin du YD et 5 ka sera comblée dans le futur avec les stalagmites Chau-stm5 et Chau-stm8 qui couvrent la première partie de l'Holocène. Les deux stalagmites Chau-stm 1 et Chau-stm4 se sont développées, quant à elles, pendant la seconde partie de l'Holocène. Leur composition isotopique est nettement plus appauvrie à la fois pour le $\delta^{18} \mathrm{O}$ et pour le $\delta^{13} \mathrm{C}$ et sont proches de la composition isotopique mesurées sur des stalactites modernes (fig. 4 et 5). Les conditions environnementales (végétation, climat) ont sans doute peu varié depuis le début de leur formation et sont proches des conditions actuelles. Cependant, on remarque une évolution vers un enrichissement dans le signal du $\delta^{18} \mathrm{O}(\sim 2 \%$ ) entre $4,6 \mathrm{ka}$ et $3,5 \mathrm{ka}$, synchrone sur les deux stalagmites; celle-ci pourrait correspondre à une légère dégradation climatique, pas suffisamment intense pour avoir changé significativement les conditions biopédologiques et donc le $\delta^{13} \mathrm{C}$.

\section{CONCLUSIONS}

Cette première étude isotopique a permis de préciser le cadre paléoenvironnemental et chronologique de la grotte Chauvet, ceci pour le Tardiglaciaire et l'Holocène. Grâce aux analyses U/Th effectuées sur les stalagmites et sur les planchers stalagmitiques, des âges minimums ont pu être donnés à l'éboulis d'entrée et à l'effondrement de la salle Hillaire. Les principales phases de croissance sont à rattacher aux variations climatiques; elles correspondent à la fin du stade isotopique 3 et surtout à la déglaciation (BA, YD et Holocène) et pourront être précisées avec de nouveau prélèvements. L'âge ${ }^{14} \mathrm{C}$ des charbons de bois trouvés sur le sol archéologique a été confirmé en plusieurs endroits avec les âges U/Th de la calcite qui les recouvrait. Enfin, les profils isotopiques effectués sur les stalagmites montrent un enregistrement paléoclimatique remarquable, comparable aux enregistrements glaciaires, marins ou lacustres dans leur résolution et leur sensibilité climatique.

Remerciements : Merci à J. Clottes pour son accueil au sein de l'équipe. Merci à J.-M. Geneste et B. Gély pour leur aide lors des campagnes d'échantillonnage dans la grotte ainsi qu'aux autres membres de l'équipe pour leurs conseils. Nous tenons aussi à remercier F. Bourges (G.E. Conseil, St Girons) qui a effectué régulièrement les prélèvements d'eau de pluie de la station d'Orgnac. Les analyses par ablation laser ont été effectuées au musée de Thervuren (J. Navez; Bruxelles) en collaboration avec E. Keppens et S. Verheyden de l'Université Libre de Bruxelles. Les analyses U/Th TIMS des échantillons Chau-stml à Chau-stm 3 ont été effectuées par V. Plagnes et C. Causse au LSCE et publiées dans Genty et al., 2004. Cette étude a été financée par le ministère de la Culture (DRAC Rhône-Alpes), le CNRS (LSCE, programmes ECLIPSE et PNEDC) et le CEA. 


\section{RÉFERENCES BIBLIOGRAPHIQUES}

BARD É., ROSTEK F., MÉNOT-COMBES G. (2004) - A better radiocarbon clock, Science, vol. 303, p. 178-179.

BAR-MATTEWS M., AYALON A., MATTEWS A., SASS E., HALICZ L. (1996) - Carbon and oxygen isotope study of the active watercarbonate system in a karstic Mediterranean cave: Implications for paleoclimate research in semiarid regions, Geochimica et Cosmochimica Acta, vol. $60, \mathrm{n}^{\circ} 2$, p. $337-347$.

BAR-MATTEWS M., AYALON A. (1997) - Late Quaternary Paleoclimate in the Eastern Mediterranean Region from Stable Isotope Analysis of Speleothems at Soreq Cave, Israel, Quaternary Research, vol. 47 , p. $155-168$.

BAUJARD S. (2000) - Peintures pariétales de Lascaux en microscopie électronique à balayage et à transmission, mémoire TER de l'université d'Évry-Val d'Essonne, Centre de Recherche et de Restauration des Musées de France, 24 p.

BEAULIEU J.-L. de, REILLE M. (1992) - Long Pleistocene pollen sequences from the Velay Plateau (Massif central, France), Veget. Hist. Archaeobot., vol. 1, p. 233-242.

BLANC J.-J. (1985) - Phases d'effondrements aux grottes préhistoriques : du Würm à l'Holocène dans le Midi de la France, Karstologia, vol. 6, p. 21-28.

BOCHERENS H., DRUCKER D., BILLIOU D., GÉLY B., GENESTE J -M., KERVAZO B., MOUSSA I. PHILIPPE M. (2003) - Grotte Chauvet : étude de la diagenèse de l'os et implications pour les études biogéochimiques (datation au radiocarbone, isotopes stables du carbone et de l'azote), Journées de la Société préhistorique française "La grotte Chauvet à Vallon-Pont-d'Arc: bilan des recherches pluridisciplinaires", Lyon, 11-12 octobre 2003.

CHAUVET J.-M., BRUNEL-DESCHAMPS É., HILLAIRE C. (1995) La grotte Chauvet, éd. Seuil, 115 p.

CLOTTES J., CHAUVET J -M. BRUNEL-DESCHAMPS É., HILLAIRE C., DAUGAS J.-P., ARNOLD M., CACHIER H., ÉVIN J. FORTIN P., OBERLIN C., TISNÉRAT N., VALLADAS H. (1995) Les peintures paléolithiques de la grotte Chauvet-Pont-d Arc à VallonPont-d'Arc (Ardèche, France) : datations directes et indirectes par la méthode du radiocarbone, $C$. R. Acad. Sci. Paris, vol. 320, série IIa, p. $1133-1140$

CORTIJO E., YIOU P., LABEYRIE L., CREMER M. (1995) - Sedimentary record of rapid climatic variability in the north-atlantic ocean during the last glacial cycle, Paleoceanography, vol. 10, $\mathrm{n}^{\circ} 5, \mathrm{p} .911$ 926

CUFFEY K.M., VIMEUX F. (2001) - Covariation of carbon dioxide and temperature from the Vostok ice core after deuterium-excess correction, Nature, vol. 412, p. 523-527.

DANSGAARD W., JOHNSEN S.J., CLAUSEN H.B., DAHI-JENSEN D. GUBDESTRUP N.S., HAMMER C.U., HVIDBERG C.S., STEFFENSEN J.P., SVEINBJÖRNSDOTTIR A.E., JOUZEL J., BOND G. (1993) - Evidence for general instability of past climate from a $250-\mathrm{kyr}$ ice-core record, Nature, vol. 364, p. 218-220.

DELANNOY J.-J., DEBARD É.. FERRIER C., KERVAZO B., PERRETTE Y. (2001) - Contribution de la cartographie morphologique souterraine dans l'étude spéléogénique de la grotte Chauvet. Premiers éléments spéléogéniques et implications paléogéographiques, préhistoriques et paléontologiques, Quaternaire, 12, p. 235-248.

DELANNOY J.-J., PERRETTE Y., DEBARD É.. FERRIER C.. KERVAZO B.. PERROUX A.-S. (2003) - Genèse el évolution de la grotte Chauvet : bilan des études karstogéniques, Journées de la Société préhistorique française "La grotte Chawet à Vallon-Pont-d Arc : bilan des recherches pluridisciplinaires", Lyon, 11-12 octobre 2003.

ESCALON DE FONTON M. (1971) - Stratigraphies, effondrements, climatologie des gisements préhistoriques du Sud de la France du Würm III à l'Holocène, $A F E Q, 4$, p. 199-207.

FERRIER C., DEBARD É., KERVAZO B., PERROUX A.-S.. DELAN. NOY J.-J.. PERRETTE Y. (2003) - Apport de l'étude des formes et des remplissages : exemple des salles Hillaire et du Crâne, Journées de la Société préhistorique française "La grotte Chauvet à VallonPont-d'Arc: bilan des recherches pluridisciplinaires ", Lyon, 11-12 octobre 2003.

FRISIA S., BORSATO A., FAIRCHILD I.J., McDERMOTT F. (2000) Calcite fabrics, growth mechanisms and environments of formation in speleothems from the Italian Alps and Southwestern Ireland, Journal of Sedimentary Research, 70, p. 1183-1196.

GENTY D., BAKER A., BARNES W., MASSAULT M. (1996) - Growth rate, grey level and luminescence of stalagmite laminae, Karst Water Institute Special Publication, 2, University of Bergen, Norway, p. 36-39.

GENTY D., QUINIF Y. (1996) - Annually laminated sequences in the internal structure of some Belgian stalagmites - Importance for paleoclimatology, Journal of Sedimentary Research, vol. 66, p. 275-288

GENTY D., DAUPHIN Y., DEFLANDRE G., QUINIF Y. (1997) Exemples de particules d'origine anthropique piégées dans les lamines de croissance de stalagmites - Intérêt pour la reconstitution des environnements humains anciens, Quaternaire, vol. 8, p. 149-157.

GENTY D., MASSAULT M., GILMOUR M., BAKER A., VERHEYDEN S., KEPPENS E. (1999) - Calculation of past dead carbon proportion and variability by the comparison of AMS ${ }^{14} \mathrm{C}$ and TIMS U/Th ages on two Holocene stalagmites, Radiocarbon, vol. 41, p. 251270 .

GENTY D., DIOT M.-F., O'YL W. (2001) - Sources of pollen in stalactite drip water in two SW-France caves, Cave and Karst Science, vol. $28, n^{\circ} 2$, p. $59-66$

GENTY D., BLAMART D., OUAHDI R., GILMOUR M., BAKER A., JOUZEL J., VAN-EXTER S. (2003) - Precise dating of DansgaardOeschger climate oscillations in Western Europe from speleothem datat, Nature, vol. 421, p. 833-837.

GENTY D., GHALEB B., PLAGNES V., CAUSSE C. VALLADAS H. BLAMART D., MASSAULT M., GENESTE J-M., CLOTTES J. (2004) - Datations U/Th (TIMS) et ${ }^{14} \mathrm{C}$ (AMS) des stalagmites de la grotte Chauvet (Ardèche, France) : intérêt pour la chronologie des événements naturels et anthropiques de la grotte. Comptes Rendus PALEOV.

GROOTES P.M., STUIVER M. WHITE J.W. ., JOHNSEN S.J. JOUZEL J. (1993) - Comparison of the oxygen isotope records from the GISP2 and GRIP Greenland ice cores, Nature, vol. 366, p. 552554.

GUIOT J., BEAULIEU J.-L. de, CHEDDADI R., DAVID F., PONEL P., REILLE M. (1993) - The climate in Western Europe during the last Glacial/Interglacial cycle derived from pollen and insect remains, Palaeogeography, Palaeoclimatology: Palaeoecology, vol. 103, p. 7393.

HENDY C.H. (1971) The isotopic geochemistry of speleothems - I The calculation of the effects of different modes of formation on the isotopic composition of speleothems and their applicability as paleoclimatic indicators. Geochimica et Cosmochimica Acta, vol. 35 , p. 801-824.

KENDALL A.C., BROUGHTON P.L. (1978) - Origin of fabrics in speleothems composed of columnar calcite crystals, Journal of Sedimentary Petrology, vol. 48, p. 519-538.

KERVAZO B. FERRIER C. DEBARD É.. DELANNOY J.-J., PERRETTE Y.. PERROUX A.-S. (2003) - Grotte Chauvet (Ardèche France) : les dépóts de la salle Hillaire et leurs relations avec les vestiges archéologiques et paléontologiques, Bulletin de la Société géologique de Frince, soumis.

KIM SANG-TAE. ONEIL J.R. (1997) - Equilibrium and nonequilibrium nxygen isotope effect in synthetic carbonates. Geochimica et Cosmochimica Acta, vol. 61, p. 3461-3475.

LIW.X. LUNDBERG J. DICKIN A.P. FORD D.C. SCHWARCZ H.P. McNUTT R.. WILLIAMS D. (1989) - High-precision mass-spectrometric uranium-series dating of cave deposits and implications for palaeoclimate studies, Nature, vol. 339. p. 534-536. 
MARTINSON D.G., PISIAS N.G., HAYS J.D., IMBRIES J., MOORE T. C., SHACKELTON N.J. (1987) - Age dating and the orbital theory of Ice Ages: development of a high-resolution 0 to 300 year chronostratigraphy, Quaternary Research, vol. 27, p. 1-29.

McDERMOTT F. (2004) - Palaeoclimate reconstructions from stable isotope variations in speleothems: a review, Quaternary Science Review, vol. 23, p. $901-918$

MENU M., WALTER P. (1995) - Analyse des peintures préhistoriques, Techné, $\mathrm{n}^{\circ} 2$.

O'NEIL J.R., CLAYTON R.N., MAYEDA T.K. (1969) - Oxygen isotope fractionation in divalent metal carbonates, Journal of Chemical Physics, vol. 51, p. 5547-5558.

PERRETTE Y., DELANNOY J.-J., GENTY D., DESTOMBES J-.L., QUINIF Y. (1997) - Enregistrement de l'activité charbonnière dans les spéléothèmes de Choranche (Vercors, France), Colloque "Milieux carbonatés continentaux", UIS, La Chaux-de-Fonds, Neuchâtel, Suisse, 4 p.

SHACKLETON N.J., HALL M.A. (2001) - Phase relationships between millennial-scale events 64,000-24,000 years ago, Paleoceanographic currents, vol. 15, p. 565-569.

SPÖTL C., MANGINI A. (2003) - Stalagmite from the Austrian Alps reveals Dansgaard-Oeschger events during isotope stage 3: Implications for the absolute chronology of Greenland ice cores, Earth and Planetary Science Letters, vol. 203, p. 507-518.

STUIVER M., KRA R.S. dir. (1986) - Calibration issue, Proceeding of the $12^{\text {th }}$ International ${ }^{14} \mathrm{C}$ conference, Radiocarbon, vol. $28, \mathrm{n}^{\circ} 2 \mathrm{~B}$, p. $805-1030$.

VALLADAS H., ClOTTES J., GENESTE J.-M., GARCIA M.-A., ARNOLD M., CACHIER H., TISNERAT-LABORDE N. (2001) Evolution of prehistoric cave art, Nature, vol. 413, p. 479.
VON GRAFENSTEIN U., ERLENKEUSER H., BRAUER A., JOUZEL J., JOHNSEN J. (1999) - A Mid-European Decacal IsotopeClimate Record from 15.500 to 5000 years B.P., Science, vol. 284, p. 1654-1657.

VOUVÉ J. (1995) - Essai de caractérisation d'objets colorants découverts dans la grotte de Lascaux (Dordogne, France) et implications futures, $I$ 'Anthropologie, $1,99, \mathrm{n}^{\circ}$ 2/3, Paris, p. 478-483.

WAELBROECK C., JOUZEL J., LABEYRIE L. (1995) - A comparison of the Vostok ice deuterium record and series from Southern Ocean core MD 88-770 over the last two glacial-interglacial cycles, Climate Dynamics, vol. 12, ${ }^{\circ} 2$, p. 113-123.

\section{Dominique GENTY}

Laboratoire des Sciences du Climat et de l'Environnement, UMR CEA/CNRS 1572, bat. 709, L'Orme des Merisiers, CEA Saclay, 91191 Gif-sur-Yvette cedex, France genty@1sce.saclay.cea.fr

Bassam GHALEB GEOTOP, UQUAM, C.P. 8888 , succ. Centre-Ville, H3C 3P8, Montréal, Canada,

Dominique BLAMART

Laboratoire des Sciences du Climat et de l'Environnement, UMR CEA/CNRS 1572, bat. 709, L'Orme des Merisiers, CEA Saclay, 91191 Gif-sur-Yvette cedex, France 